\title{
Understanding Protein Arginine Methyltransferase 1 (PRMT1) Product Specificity from Molecular Dynamics
}

\author{
Symon Gathiaka ${ }^{\ddagger}$, Brittany Boykin ${ }^{\ddagger}$, Tamar Cáceres ${ }^{\dagger}$, Joan M. Hevel ${ }^{\dagger}$, and Orlando \\ Acevedo*** \\ ${ }^{\ddagger}$ Department of Chemistry, University of Miami, Coral Gables, Florida 33146. ${ }^{\dagger}$ Department of \\ Chemistry and Biochemistry, Utah State University, Logan, Utah 84322. \\ E-mail: orlando.acevedo@miami.edu
}

Submitted May 19, 2016

Abstract: Protein arginine methyltransferases (PRMTs) catalyze the post-translational methylation of specific arginyl groups within targeted proteins to regulate fundamental biological responses in eukaryotic cells. The major Type I PRMT enzyme, PRMT1, strictly generates monomethyl arginine (MMA) and asymmetric dimethylarginine (ADMA), but not symmetric dimethylarginine (SDMA). Multiple diseases can arise from the dysregulation of PRMT1, including heart disease and cancer, which underscores the need to elucidate the origin of product specificity. Molecular dynamics (MD) simulations were carried out for WT PRMT1 and its M48F, H293A, H293S, and H293S-M48F mutants bound with S-adenosylmethionine (AdoMet) and the arginine substrate in an unmethylated or methylated form. Experimental sitedirected mutagenesis and analysis of the resultant products were also performed. Two specific PRMT1 active site residues, Met48 and His293, have been determined to play a key role in dictating product specificity, as: 1) the single mutation of Met48 to Phe enabled PRMT1 to generate MMA, ADMA, and a limited amount of SDMA; 2) the single mutation of His293 to Ser formed the expected MMA and ADMA products only; whereas 3) the double mutant H293SM48F-PRMT1 produced SMDA as the major product with limited amounts of MMA and ADMA. Calculating the formation of near-attack conformers resembling $\mathrm{S}_{\mathrm{N}} 2$ transition states leading to either the ADMA or SDMA products finds that Met48 and His293 may enable WT 
PRMT1 to yield ADMA exclusively by precluding MMA from binding in an orientation more conducive to SDMA formation, i.e., the methyl group bound at the arginine $\mathrm{N}_{\eta 2}$ position.

Abbreviations: PRMT1, protein arginine methyltransferase 1; MMA, monomethylarginine; ADMA, asymmetric dimethylarginine; SDMA, symmetric dimethylarginine; AdoMet, $S$ adenosylmethionine; AdoHcy, S-adenosyl-L-homocysteine; GGRGG, Gly-Gly-Arg-Gly-Gly; NAC, near-attack conformer.

\section{Introduction}

Protein arginine methylation has emerged as a major mechanism for regulating protein function in eukaryotic cells, e.g., signal transduction, RNA processing, gene transcription, and cellular transport function. ${ }^{1-10}$ Given the major impact of this posttranslational modification upon human diseases, particularly in cardiovascular diseases ${ }^{11,12}$ and cancer $^{13-15}$, establishing how protein arginine methyltransferases (PRMTs), the family of enzymes responsible for catalysis, dictates product formation is of significant interest. Nine human PRMT isoforms are capable of producing monomethylarginine (MMA), asymmetric dimethylarginine (ADMA), and symmetric dimethylarginine (SDMA) on targeted proteins (Figure 1). Type I PRMTs (such as PRMT1) represent the majority and exclusively form MMA and/or ADMA through the transfer of the methyl group from $S$-adenosylmethionine (AdoMet) to the guanidinium moiety of arginine residues in proteins. ${ }^{16,17}$ Type II PRMTs also produce MMA, but differ from Type I PRMTs by strictly forming the biologically distinct SDMA product in the second round of turnover. ${ }^{18}$ Type III enzymes solely generate MMA. ${ }^{19}$ 


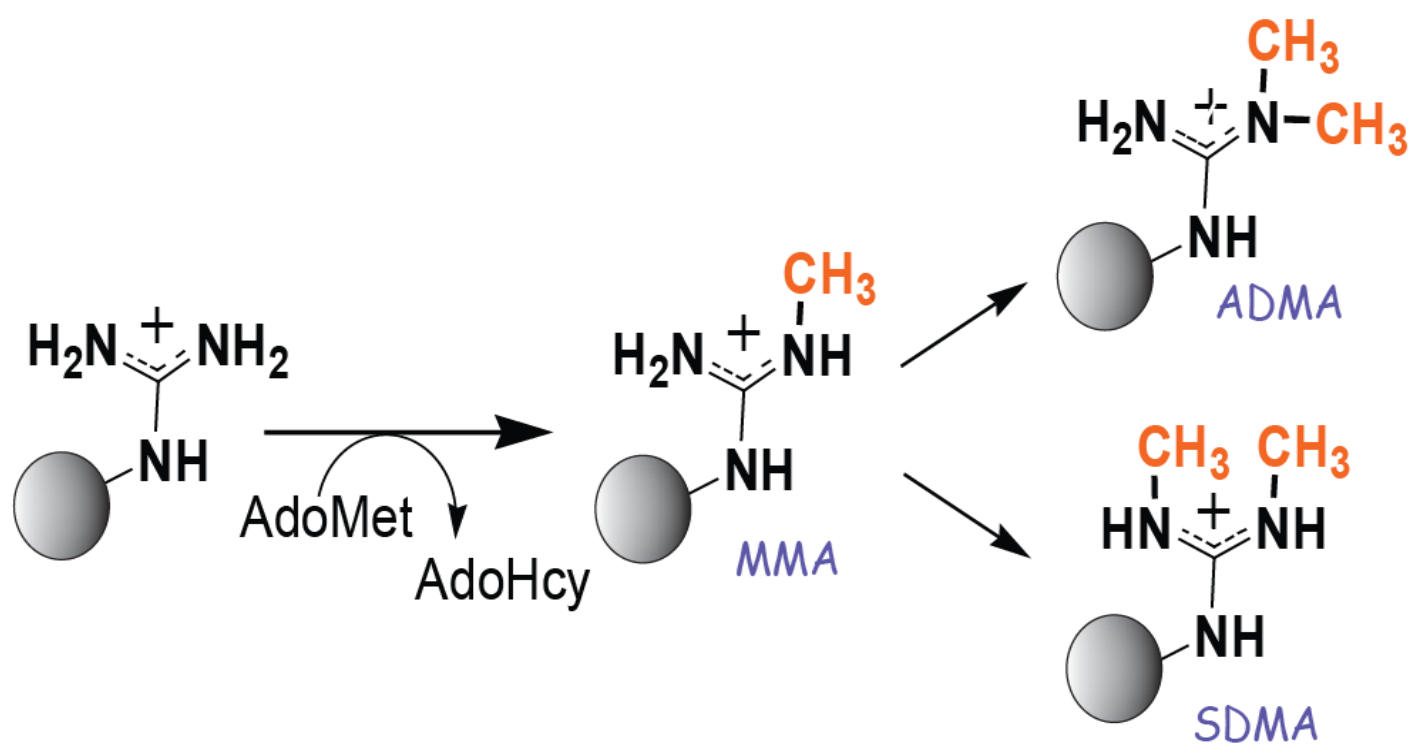

Figure 1. Methylation reactions catalyzed by PRMTs.

Different cellular responses are induced by each distinct methylated arginine, that is, MMA, ADMA and SDMA. ${ }^{20-23}$ For example, control of gene transcription by the specific formation of either MMA (silencing) or ADMA (activating) at Arg-2 on histone H3 illustrates the significance of specifying which of the three methylated arginines is formed. ${ }^{20}$ It follows that controlling the type of methylation, the amount of methylation in various patterns, and what proteins are methylated, is necessary for a healthy cell. ${ }^{24}$ However, it remains unclear how PRMTs govern the product specificity yielding symmetric and asymmetric dimethylarginine.

A crystal structure of PRMT1 was first determined by Zhang et al. in complex with the reaction product $S$-adenosyl-L-homocysteine (AdoHcy) and a 19 residue R3 peptide substrate containing three arginines. ${ }^{25}$ The monomeric protein was determined to contain four major regions: N-terminal, AdoMet/substrate arginine binding site, the $\beta$-barrel, and the dimerization arm. The whole protein is an oligomer with a hydrophobic dimer interface between the 
dimerization arm of one monomer and the AdoMet binding site of another monomer. Both distributive $^{26}$ and semi-processive ${ }^{27}$ mechanisms have been discussed for the methylation of PRMT1 substrates. Although confusing at first, Gui and co-workers showed that the identity of the substrate can influence the degree to which the substrate is processively dimethylated. ${ }^{28} \mathrm{~A}$ substrate-governed semi-processive mechanism is consistent with the presence of both monomethyl and dimethylated PRMT1 targets in vivo.

Based on evaluation of the aforementioned PRMT1 crystal structure, Rust et al. proposed an $\mathrm{S}_{\mathrm{N}} 2$ catalytic mechanism for the methyl transfer reaction between AdoMet and the argininecontaining substrate that results in the formation of a dication intermediate (Scheme 1). ${ }^{29}$ The stepwise mechanism was followed by a proton abstraction via Glu144. Mixed quantum and molecular mechanical (QM/MM) mechanistic studies on Type I enzymes, i.e., PRMT1 and PRMT3, provided computational support for the proposed mechanism. ${ }^{30,31}$ However, it is possible that two alternative residues, Glu153 or His293, could act as the active site base instead. $^{32}$ In the crystal structure, the nearby His 293 residue is $>6 \AA$ from the substrate guanidinium, a distance deemed too far for direct proton abstraction. ${ }^{29}$ Mutagenesis analysis and a lack of solvent isotope effects (SIE) also disfavor His293 as the active site base. ${ }^{29}$ Instead, His293 has been hypothesized to form a salt bridge with a conserved residue Asp51, thereby playing a critical role in forming the two-helix boundary that separates the AdoMet and peptide binding portions of the active site. Interestingly, the same mutagenesis study reported a more dramatic effect on the reaction rate for Glu153 than Glu144, i.e., $k_{\text {cat }}$ values decreased by 140fold for G153A compared to 55-fold for G144A, suggesting a key role for Glu153 in catalysis. ${ }^{29}$ 


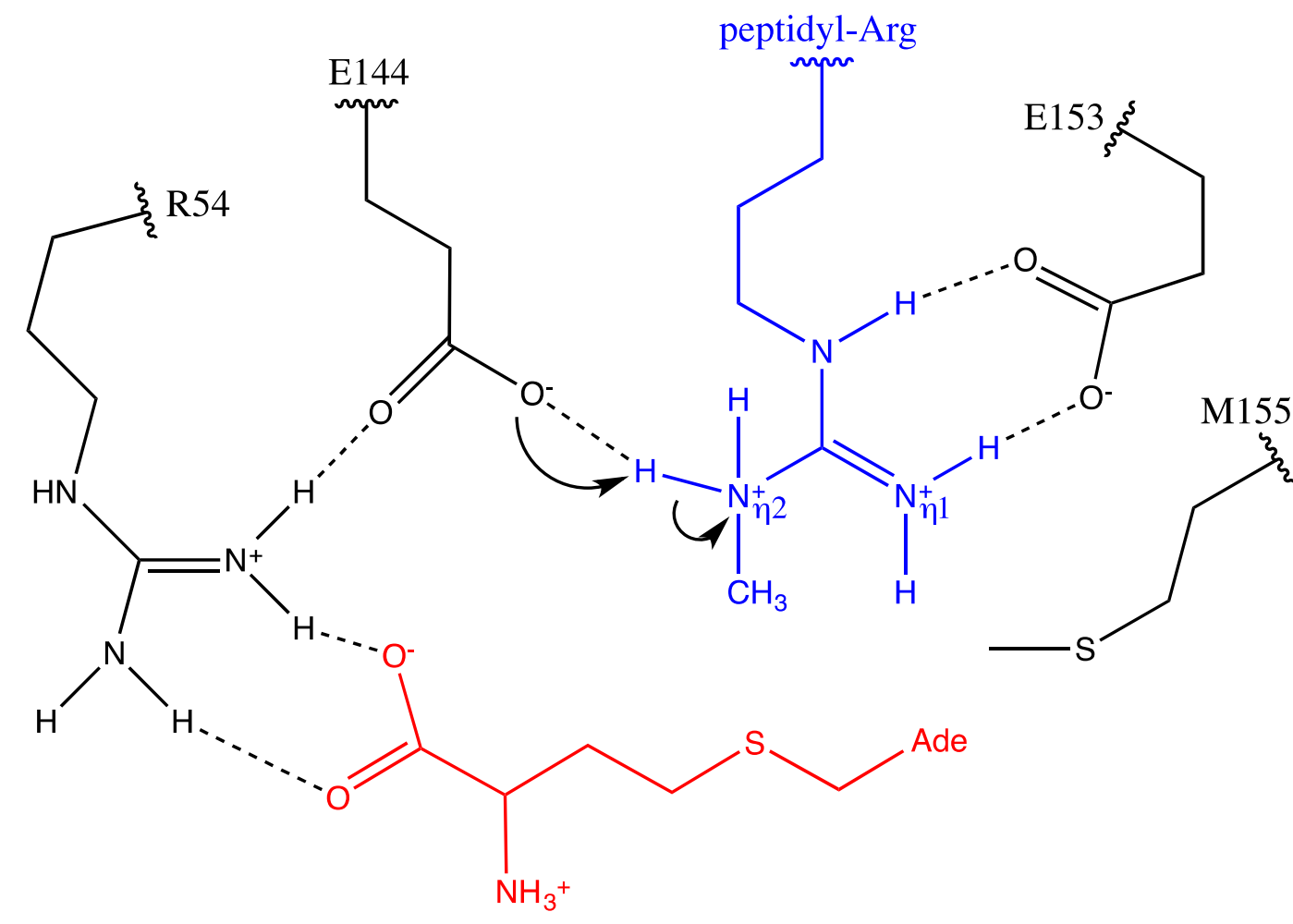

Scheme 1. Proton abstraction via Glu144 of the dication intermediate formed after the proposed $\mathrm{S}_{\mathrm{N}} 2$ methyl transfer reaction between AdoMet and the arginine-containing substrate. ${ }^{29}$

Two conserved active site residues, Met48 and Met155, have been identified to play a significant role in enzymatic activity and regulation of mono- versus asymmetric dimethylation. ${ }^{33}$ Although Met48 and Met155 was suggested to govern formation of ADMA over SDMA, mutations to smaller residues Ala and Leu somewhat impaired monomethylation and greatly impaired asymmetric dimethylation, but did not change the dimethylation product. Instead, a larger substitution, Met48 to phenylalanine, produced a very small amount of SDMA. ${ }^{34}$ These experiments were complimented with computational studies to reveal an energetic cost to making SDMA that is greater than making ADMA. In addition to offering an explanation for the limited synthesis of SDMA by the remodeled PRMT1, the high-energy 
barrier for synthesizing SDMA may explain the low to absent dimethylation activity that is observed in recombinant PRMT5 enzymes (make MMA and SDMA in vivo) and suggest that other protein factors may be necessary to overcome this barrier.

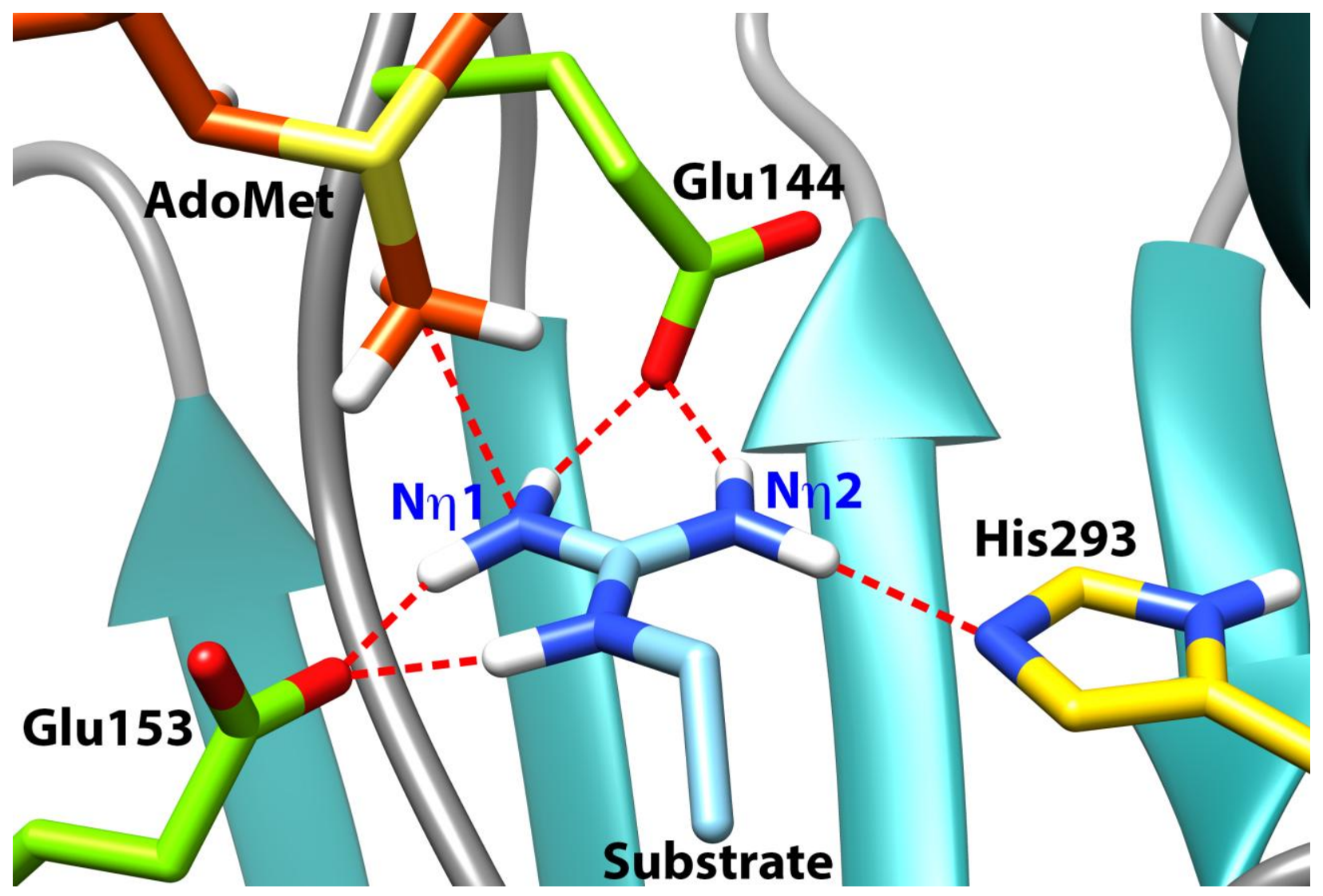

Figure 2. The GGRGG substrate bound in the PRMT1 active site where the methyl group can chemically transferred to either the $\mathrm{N}_{\eta_{1}}$ or $\mathrm{N}_{\eta_{2}}$ guanidino nitrogen on arginine.

To further explore the origin of product specificity in PRMT1, molecular dynamics (MD) simulations have been performed in this study for the WT, M48F, H293A, H293S, and H293SM48F PRMT1 enzyme systems bound with AdoMet and (1) a Gly-Gly-Arg-Gly-Gly (GGRGG) 
substrate or (2) a monomethylated GGRGG substrate where the methyl group is either covalently bound to the $\mathrm{N}_{\eta 1}$ nitrogen (MMA- $\mathrm{N}_{\eta 1}$ ) or the $\mathrm{N}_{\eta 2}$ nitrogen (MMA-N $\mathrm{N}_{\eta 2}$ ), see Figure 2. In all, 15 different bound enzyme complexes were simulated for over $4.5 \mu$ s of total calculation time. Extensive analysis has been conducted to help elucidate the origin of residue influence upon product specificity. Experimental mutagenesis studies were performed for the H293S and H293S-M48F PRMT1 mutants and product formation was analyzed using reverse phase (RP)HPLC. This joint computational and experimental study further clarifies the role of specific residues within the PRMT1 active site that dictate product specificity.

\section{Methods}

2.1 Computational enzyme preparation. Initial Cartesian coordinates for the rat PRMT1 systems were generated from a $2.35 \AA$ resolution crystal structure (PDB ID: 1OR8). ${ }^{25}$ The reported structure contains residues 41-353 per monomer, as no clear density was available for N-terminal region that supplies residues to the active site. Missing residues $26-40$ were constructed using a comparative modeling program MODELLER 9.10..$^{35}$ A standard Protein BLAST was performed using the position specific iteration-basic local alignment search tool (PSI-BLAST) $^{36}$ against the PDB database ${ }^{37}$ using the default general algorithm parameters. Only the PRMT3 structure (PDB ID: 1F3L), ${ }^{32}$ with a query coverage of $96 \%$, possessed the absent Nterminal residues that were used as the template to append the 15 residues to the PRMT1 structure. In addition, the PRMT3 1F3L crystal structure was used to re-orient Glu153 in the same direction as $\mathrm{PRMT}^{32}$ and CARM1 ${ }^{38}$ (Supplemental Figure S1), allowing for hydrogen bonding with the substrate. The quality of the final PRMT1 structure was extensively evaluated 
using Procheck, ${ }^{39}$ ProSA $^{40}$, and Ramachandran plots (Supporting Information Supplemental Figures S2 and S3).

Apart from the arginine that undergoes methylation, coordinates for the remainder of the R3 peptide substrate was not sufficiently resolved. In order to extend the substrate in the active site, the software $\mathrm{BOMB}^{41}$ was used to insert Gly residues on both sides of the crystallographically resolved Arg. The peptide ligand Gly-Gly-Arg-Gly-Gly (GGRGG) was grown into the active site region of PRMT1 bound to AdoMet based on a superposition with existing coordinates.

2.2 MD protocol. Molecular dynamics simulations were performed on WT and mutant PRMT1 in 15 different combinations of enzyme/substrates. In each case, PRMT1 was simulated as a monomer. The resulting structures were fed into the leap module of the AMBER 12 package where the appropriate hydrogen atoms were added. The ternary systems were solvated in an box of TIP3 $\mathrm{P}^{42}$ water molecules extending at least $10 \AA$ beyond the protein and sodium ions were added to maintain charge neutrality. The ff99SB force field ${ }^{43}$ was used to construct the topology files for the protein, while the parameters for AdoMet were obtained from the generalized AMBER force field (GAFF). ${ }^{44}$

For each system, the initial structure was conjugate gradient (CG) minimized for 200 steps for the water molecules only, followed by 10,000 steps of CG optimization of the entire system to remove any bad contacts. After minimization, the full system was gradually heated from 0 to $300 \mathrm{~K}$ using a constant NVT ensemble for $50 \mathrm{ps}$ of MD using the weak-coupling algorithm with a temperature coupling value of $2.8 \mathrm{ps}$. The system was then switched to a constant NPT ensemble at $300 \mathrm{~K}$ and 1 atm using a coupling value of $2.0 \mathrm{ps}$ for both temperature and pressure and ran for 500 ps. Finally, the system was returned to NVT and equilibrated for an 
additional 500 ps. Following equilibration, $300 \mathrm{~ns}$ of production data was collected at constant NVT for each protein complex using the GPU-accelerated version of AMBER $12 .^{45}$ The coordinates and energy data were saved every 5,000 steps ( 5 ps) and 10,000 steps (10 ps), respectively. All MD simulations utilized the particle mesh Ewald method to compute the longrange Coulomb force, the SHAKE algorithm ${ }^{46}$ to restrict all covalent bonds involving hydrogen atoms, periodic boundary conditions with a nonbonded cutoff distance of $12 \AA$, and a time step of 1.0 fs. Clustering, hydrogen bond analysis, root-mean square fluctuation (rmsf), and rootmean square deviation (rmsd) calculations were computed using the ptraj and cpptraj programs within AmberTools. ${ }^{47}$ All enzyme figures were made using Chimera. ${ }^{48}$

2.3 Binding energy calculations. The AMBER suite provides a Molecular Mechanics/Poisson-Boltzmann Surface Area (MM/PBSA) calculation utility ${ }^{49}$ that can be used to estimate binding affinity starting with a molecular dynamics trajectory. The binding free energy is expressed as:

$$
\Delta G_{\text {binding }}=\Delta G_{\text {complex }}-\Delta G_{\text {receptor }}-\Delta G_{\text {ligand }}
$$

Where the free energy change associated with the complex, the unbound receptor and ligand are approximated as:

$$
\Delta G_{\text {solvated }}=E_{\text {gas }}+\Delta G_{\text {solvation }}-T \Delta S_{\text {solute }}
$$

$E_{\text {gas }}$ is the potential energy (MM) from the AMBER force field, $\Delta G_{\text {solvation }}$ is the solvation free energy computed using an implicit solvent model (PBSA) and the entropic contribution, $S$, are estimated. The $\Delta G_{\text {solvation }}$ is the sum of the electrostatic and nonpolar contributions, with the nonpolar portion derived using the solvent accessible surface area (SASA). ${ }^{49}$ The dielectric constant for the implicit solvent and the interior of the protein was 80 and 1, respectively. In this 
work, free energies were determined by using all the snapshots from the 300 ns trajectories (taken every 5ps) for the respective complexes. The necessary ensembles for the bound and unbound states for the receptor and ligand were extracted using the single trajectory protocol. Default AMBER values in the MM/PBSA program were used with the relative changes in configurational entropies assumed to be negligible and therefore the entropic contribution not included in estimating the binding free energies.

2.4 Expression and purification of PRMT1 mutant proteins. PRMT1 mutant proteins were generated using the QuikChange ${ }^{\circledR}$ site-directed mutagenesis kit (Stratagene) with sets of complementary oligonucleotide primers spanning the desired site of mutation. For each PCR, the pET28b vector (Novagen) containing the gene that codes for N-terminal histidine-tagged rat WT-PRMT1 plasmid (pET28b-PRMT1) was used as a template. Desired mutations were confirmed through DNA sequencing. Mutated proteins were purified using the same methods used to express and purify wild-type His-PRMT1. Purified proteins were more than $95 \%$ pure by SDS-PAGE.

2.5 Reverse phase-HPLC analysis of methylated arginines. The three methylated arginine products (MMA, ADMA, and SDMA) were identified as described previously. ${ }^{34}$ Briefly, assays containing $3 \mu \mathrm{M}$ WT or mutant PRMT1 proteins, $2.67 \mathrm{uM}^{3} \mathrm{H}$ labeled AdoMet + $6.9 \mathrm{uM}$ unlabeled AdoMet, $10 \mathrm{nM}$ AdoHcy nucleosidase (MTAN), and $50 \mathrm{mM}$ sodium phosphate buffer (pH 7.5) were incubated at $37{ }^{\circ} \mathrm{C}$ for 2 min. Reactions were initiated with 200 $\mu \mathrm{M}$ RKK (Acetyl GGRGGFGGKGGFGGKW) peptide and were terminated after $3 \mathrm{~h}$ with $10 \%$ (v/v, final concentration) trifluoroacetic acid (TFA) and incubated at $4^{\circ} \mathrm{C}$ for 10 minutes. TFA precipitated protein was removed through centrifugation and the supernatant (containing the peptide) was added to a glass vial containing $11 \mathrm{M} \mathrm{HCl}$. Vials were sealed and heated to $110{ }^{\circ} \mathrm{C}$ 
overnight for acid hydrolysis. Hydrolyzed amino acids from the enzyme reactions were filtered using a Durapore ${ }^{\circledR}$-PVDF $0.65 \mu \mathrm{m}$ centrifugal filter. The amino acids were derivatized using $o$ phthaldialdehyde and separated with a Gemini $5 \mu \mathrm{m} \mathrm{C18} \mathrm{110- \AA} \mathrm{LC} \mathrm{column} \mathrm{250x} 4.6 \mathrm{~mm}$ (Phenomenex). Mobile phase A consisted of $40 \mathrm{mM}$ sodium phosphate buffer, $\mathrm{pH} 7.8$, and mobile phase B was acetonitrile/methanol/ $\mathrm{H}_{2} \mathrm{O}(45: 45: 10$, v/v). Fractions were collected, and radioactivity was counted in $5 \mathrm{ml}$ of scintillation mixture (Fisher). MMA, ADMA, or SDMA standard amino acids were used to verify the identity of the methylated products generated.

\section{Results and Discussion}

To evaluate the structural changes that occur within the active site of PRMT1 when bound to the various GGRGG substrates in their respective methylated arginine states, MD simulations were performed and analyzed. For simplicity, the GGRGG substrate will be referred to herein as Arg; WT-Arg denotes the WT PRMT1 enzyme bound with GGRGG and AdoMet. The monomethylated GGRGG substrate will be denoted as MMA- $\mathrm{N}_{\eta 1}$ when the methyl group is covalently bound at the $N_{\eta 1}$ nitrogen and MMA- $N_{\eta 2}$ when bound to the $N_{\eta 2}$ nitrogen (see Figure 2 for substrate orientation within the active site). The root-mean-square deviation (rmsd) and the root-mean-square fluctuations (rmsf) of the trajectories were calculated for the WT and mutant PRMT1 systems in order to monitor the structural stability and global conformational changes of each simulation. The rmsd values of the backbone protein atoms are provided in the Supporting Information (Supplemental Figure S4) and yielded steady average values of approximately $3.5 \AA$ over 300 ns for all complexes, confirming stable systems. 


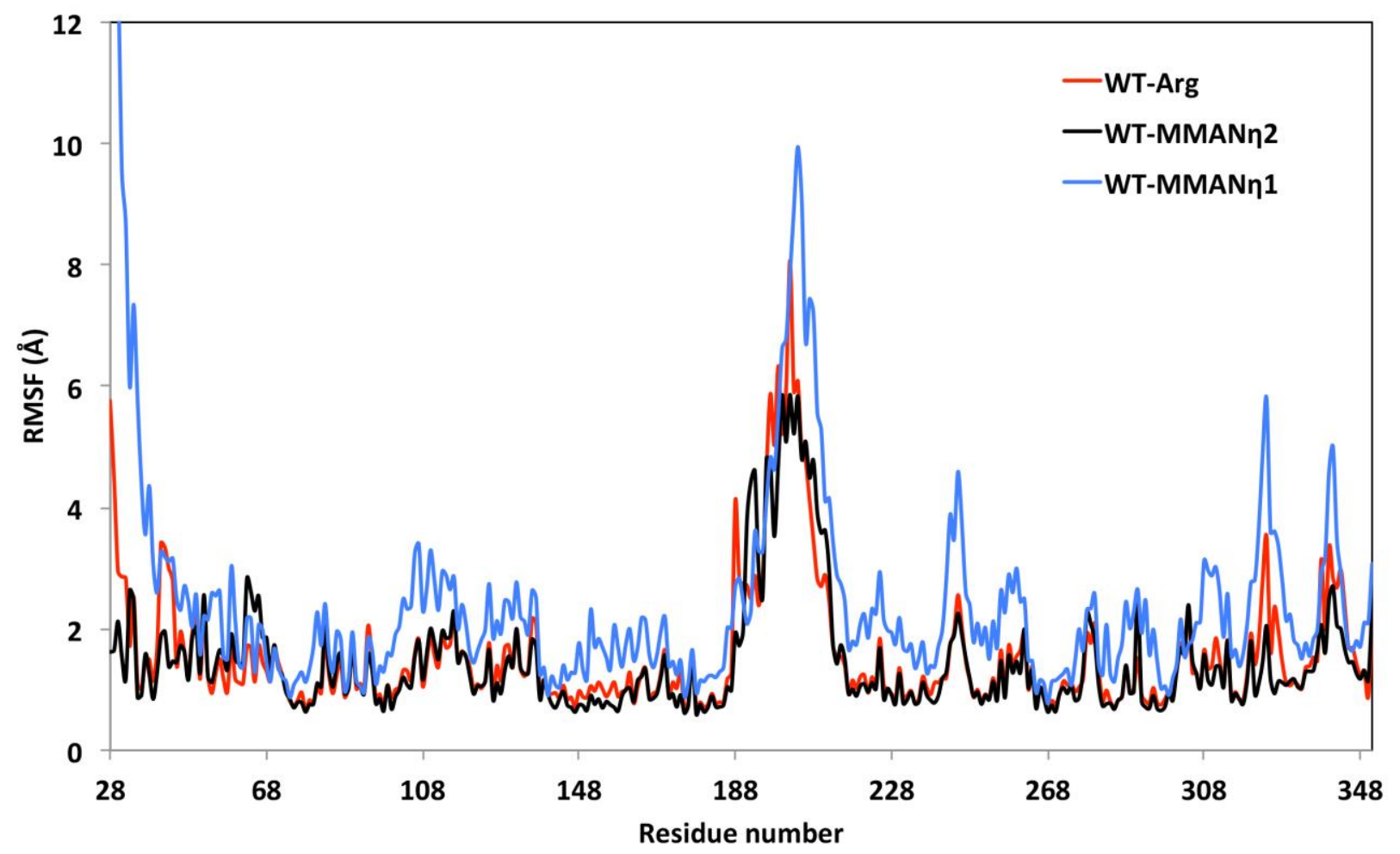

Figure 3. Root-mean-square fluctuations (RMSF) by residue of the WT-PRMT1 backbone atoms $(\mathrm{C}, \mathrm{C} \alpha$ and $\mathrm{N})$ relative to the average structure.

Rmsf calculations allow for clearer insight into PRMT1 dynamics and fluctuations in response to substrate binding. Figure 3 shows the positional fluctuations of the WT-Arg, WTMMA-N ${ }_{\eta 1}$, and WT-MMA-N ${ }_{\eta 2}$ PRMT1 simulations. Rmsf plots for the PRMT1 mutants are similar to WT and are provided in Supplemental Figure S5. Visual inspection of the rmsf graph finds a large peak encompassing the residues Arg188 to Lys215. This area of protein corresponds to a highly flexible dimerization arm composed of three helices linked by a loop: $\alpha$ helix 188-194, $3_{10}$-helix 196-199, and $\alpha$-helix 210-214. The extensive conformational changes computed suggest that Asp187 and Glu216 may act as hinges. However, it is important to note 
that the enzyme is active as a dimer, but the current simulations are on a monomeric unit. Recent simulations of a PRMT1 dimer have shown that conformational fluctuations in the dimerization arm are stabilized upon dimerization. ${ }^{50}$ The AdoMet binding site residues (28-145) were also found to be modestly flexible. The first two helices (43-63) of the N-terminus lie at the entrance of the binding site and fluctuate appreciably as they are expected to open and close for substrate binding and product release. The predicted flexibility of this $\mathrm{N}$-terminal region correlates with the lack of clear electron density in the reported crystal structure. ${ }^{25}$

Lowering the activation barrier through preorganization of the active site into appropriate electrostatic interactions that stabilize the transition state is the most significant factor affecting enzymatic catalysis and likely product specificity. ${ }^{51}$ Other factors may also contribute, such as the thermodynamic stabilization of near-attack conformations (NACs) resembling the transition state. ${ }^{52}$ NACs have been characterized by Bruice as possessing a reacting atoms distance within $3.2 \AA$ and an approach angle of $\pm 15^{\circ}$ of the bonding angle in the transition state for reactions involving $\mathrm{O}, \mathrm{N}, \mathrm{C}$ and $\mathrm{S}$ atoms. ${ }^{53}$ In the current study, geometric orientations of the substrates in the various ternary systems were explored over the entire simulation to determine the probability of sampling near the $\mathrm{S}_{\mathrm{N}} 2$ transition states leading to the formation of either MMA, ADMA, or SDMA products. The preferred orientations by the substrate over time were evaluated by comparing the distributions of $d+0.5(\cos \theta)$ for all trajectories, where $d$ is the attack distance between the nitrogen atom in arginine $\left(\mathrm{N}_{\eta 1}\right.$ or $\left.\mathrm{N}_{\eta 2}\right)$ and the methyl carbon in AdoMet, and $\theta$ is the respective attack angle $\left(\mathrm{N} \cdots \mathrm{CH}_{3} \cdots \mathrm{S}\right)$. The most ideal $\mathrm{S}_{\mathrm{N}} 2$ attack angle of $\theta=\pi$ radians $\left(180^{\circ}\right)$ will favorably scale the down the final $d$ value by -0.5 , i.e., $0.5(\cos \pi)$. Therefore the scaling rewards a productive $\mathrm{S}_{\mathrm{N}} 2$ angle between $90-180^{\circ}$ and penalizes angles $<90^{\circ}$. 
3.1 WT PRMT1. Investigation of the WT PRMT1 enzyme bound with AdoMet and the GGRGG substrate (i.e., WT-Arg) finds no appreciable difference between the probabilities of forming an NAC to yield the MMA product with the methyl group bound at either the $\mathrm{N}_{\eta 1}$ or $\mathrm{N}_{\eta 2}$ atom (Figure 4). Average $d+0.5(\cos \theta)$ distances were computed to be 3.4 and $3.6 \AA$ between the reactive AdoMet methyl-C atom and the $\mathrm{N}_{\eta 1}$ and $\mathrm{N}_{\eta 2}$ atoms, respectively (Figure 5). Favorable electrostatics via residues Glu144, Glu153, and His293 play a large role in localizing the GGRGG substrate for the subsequent $\mathrm{S}_{\mathrm{N}} 2$ methylation reaction (Figure 6). Hydrogen bonding was investigated by computing percentages from the populations established over the course of the trajectory with a distance cutoff of $3 \AA$ between the heavy atoms and a cutoff angle of 135 degrees between the donor hydrogen and acceptor $\mathrm{O}$ or $\mathrm{N}$ atoms. The percent occupancy of the hydrogen bonds formed between the carboxylate oxygen atoms of Glu144 (94.5\% and 53.2\%) and Glu153 (92.2\% and 62.6\%) and the hydrogen atoms bound to the $\mathrm{N}_{\eta 1}$ and $\mathrm{N}_{\eta 2}$ atoms of the Arg substrate were steadily high throughout the entire simulation (Figure 7 and Table 1). These key electrostatic interactions are critical in regard to the positioning of the substrate and for increasing the nucleophilicity of the guanidino moiety for the methylation reaction, an otherwise poor nucleophile. For example, Thompson and coworkers reported that a single mutation of Glu153 in PRMT1 to an aspartate residue reduced the $k_{\text {cat }}$ value by 31 -fold, emphasizing that even a subtle difference in active site geometry, i.e., an extra methylene in the catalytic residue, has a large effect upon substrate orientation and/or nucleophilic activation. ${ }^{29}$ 
Table 1. Hydrogen bonding percent occupancy (\%) (acceptor-donor) involving unmethylated and methylated GGRGG substrates and the active site residues Glu144, Glu153, His293, Arg54, Tyr39, and Asp51 over the entire simulation for the WT, M48F, and H293S-M48F PRMT1 systems.

\begin{tabular}{|c|c|c|c|c|c|c|c|}
\hline \multirow{3}{*}{ H-acceptor } & \multirow[b]{3}{*}{ H-donor ${ }^{a}$} & \multicolumn{2}{|c|}{ WT } & \multicolumn{2}{|c|}{$\mathrm{M} 48 \mathrm{~F}$} & \multicolumn{2}{|c|}{ H293S-M48F } \\
\hline & & Arg & MMA-N $N_{\eta 1}$ & Arg & 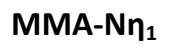 & Arg & MMA- $\mathbf{N}_{\mathrm{n} 2}$ \\
\hline & & \multicolumn{6}{|c|}{ \%occupancy } \\
\hline Glu144@OE1+OE2 & Arg@HH12 & 94.5 & 55.1 & 94.3 & 17.1 & 16.3 & 85.62 \\
\hline Glu144@OE1+OE2 & Arg@HH22 & 53.2 & N/A & 74.1 & N/A & 22.1 & 68.1 \\
\hline Glu153@OE1+OE2 & Arg@HE & 92.2 & 83.8 & 65.4 & 68.3 & 20.3 & 27.9 \\
\hline Glu153@OE1+OE2 & Arg@HH21 & 62.6 & 81.6 & 25.1 & 85.5 & 20.4 & 21.2 \\
\hline His293@NE2 & Arg@HH11 & 60.4 & 49.0 & 61.0 & 25.2 & $\mathrm{~N} / \mathrm{A}$ & N/A \\
\hline Glu144@OE1+OE2 & Arg54@HH11 & 78.5 & 75.5 & 91.7 & 83.8 & 68.0 & 95.0 \\
\hline Glu153@OE1+OE2 & Tyr39@HH & 92.7 & 97.8 & 90.8 & 76.7 & 22.0 & 95.2 \\
\hline Asp51@OD1+OD2 & His293@HD1 & 81.3 & 87.1 & 84.3 & 83.5 & N/A & N/A \\
\hline
\end{tabular}

aGGRGG substrates are labeled here as "Arg" for all methylated and unmethylated forms. 


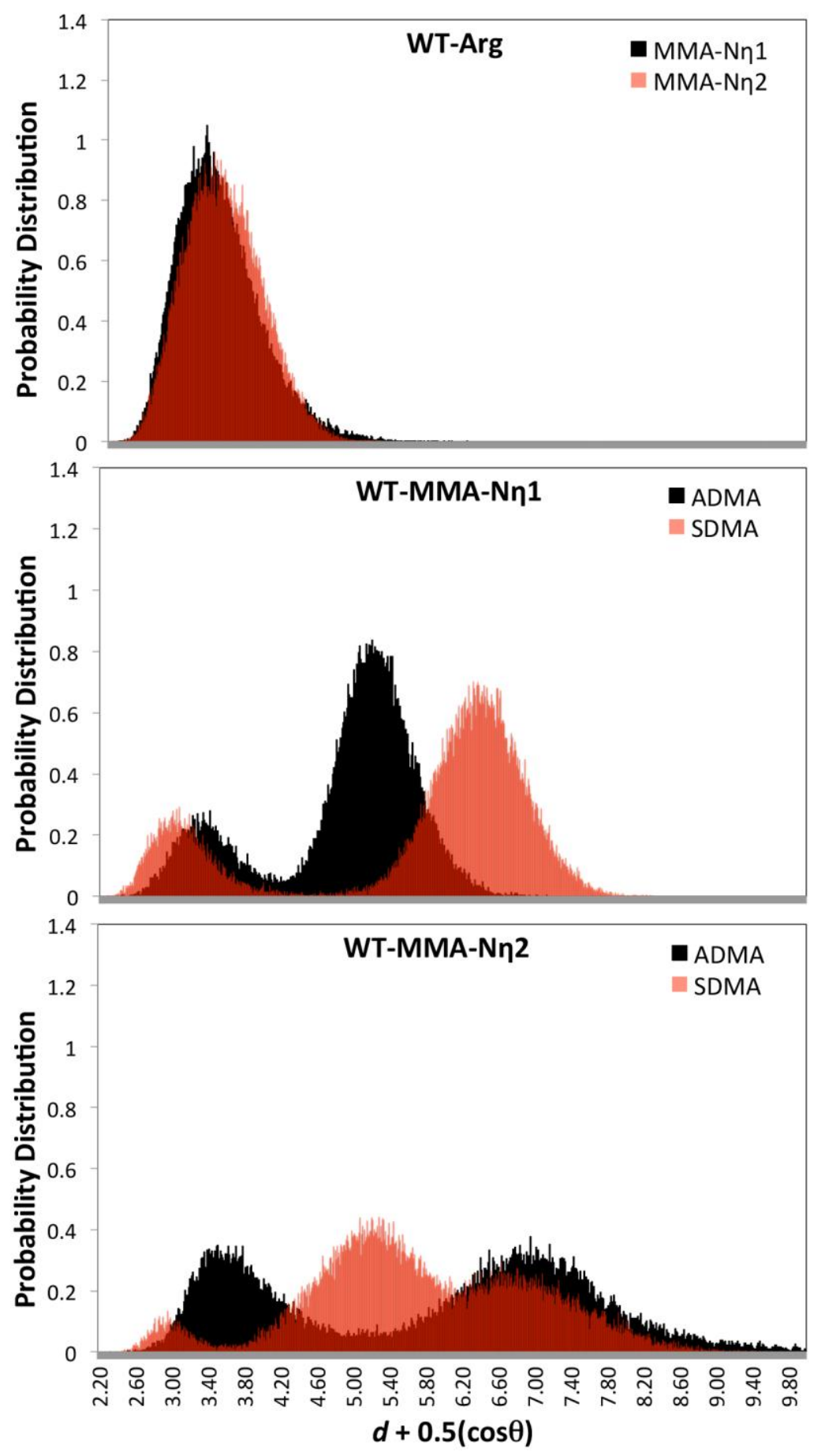

Figure 4. Probability of sampling near-attack conformers in the WT-PRMT1 complex that leads to the formation of MMA, ADMA, or SDMA products. The preferred orientations by the substrate over time were evaluated by comparing the distributions of $d+0.5(\cos \theta)$ for all 
trajectories, where $d$ is the attack distance between the nitrogen atom in arginine $\left(\mathrm{N}_{\eta 1}\right.$ and $\left.\mathrm{N}_{\eta 2}\right)$ and the methyl carbon in AdoMet, and $\theta$ is the respective attack angle $\left(\mathrm{N}^{\prime} \mathrm{CH}_{3} \cdots \mathrm{S}\right)$.

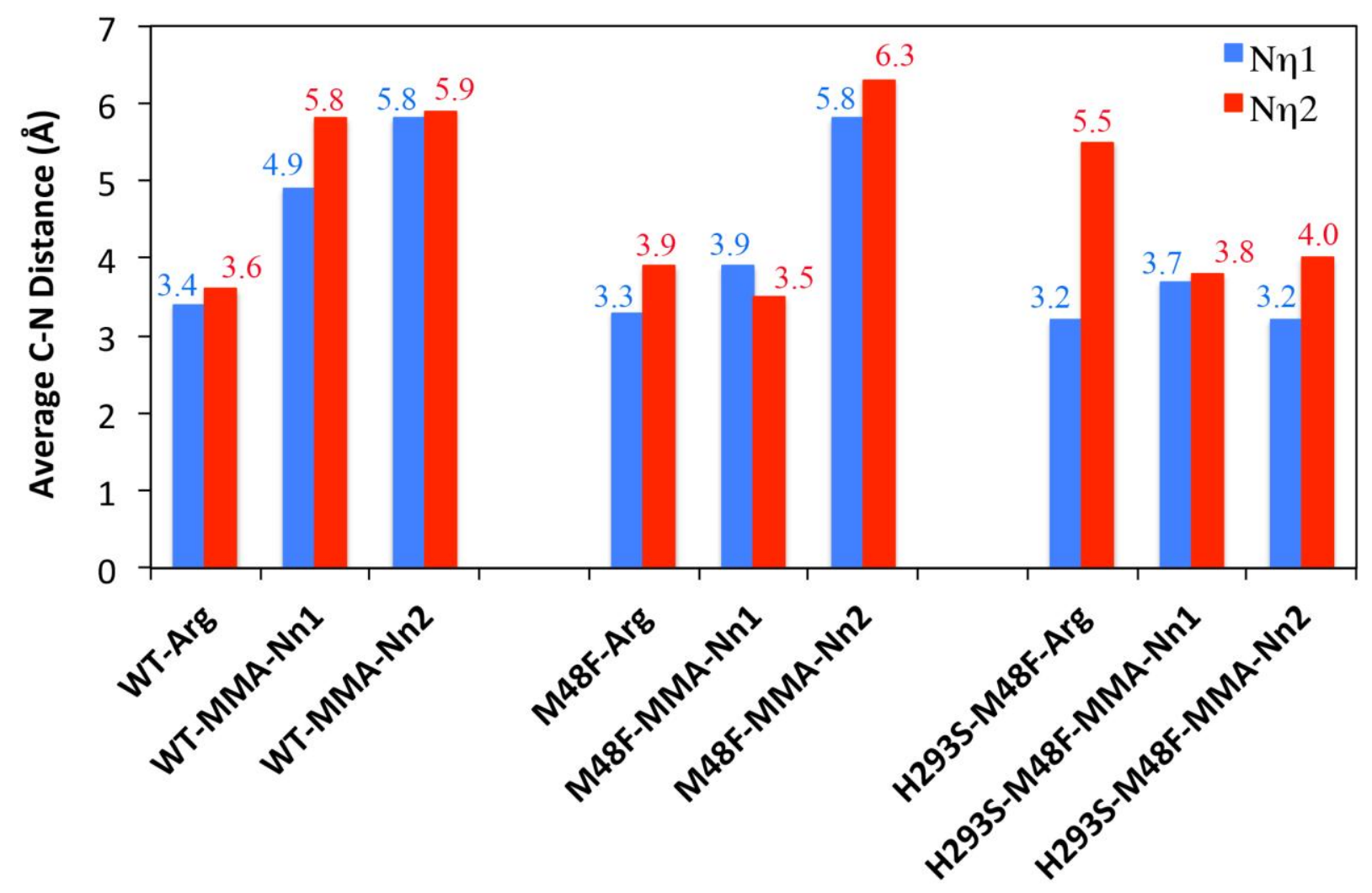

Figure 5. The average distance $(\AA)$ calculated using $d+0.5(\cos \theta)$ between the arginine $\mathrm{N}_{\eta 1}$ or $\mathrm{N}_{\eta 2}$ atom and the reacting carbon on the AdoMet methyl group for the WT, M48F, and H293SM48F PRMT1 enzymes. 


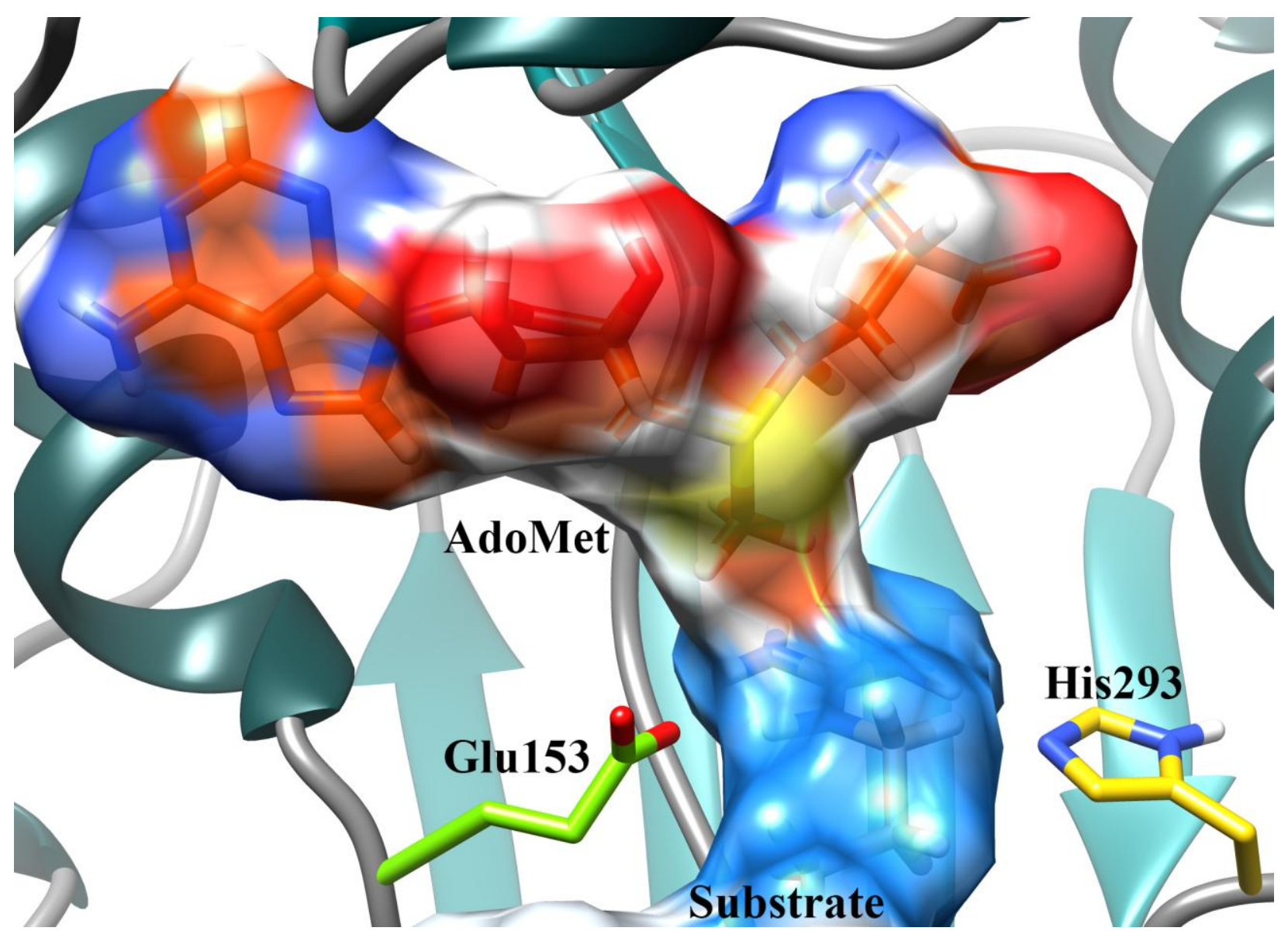

Figure 6. Glu153 and His293 localize the GGRGG substrate in the PRMT1 active site through hydrogen bonding for subsequent methylation via AdoMet. 


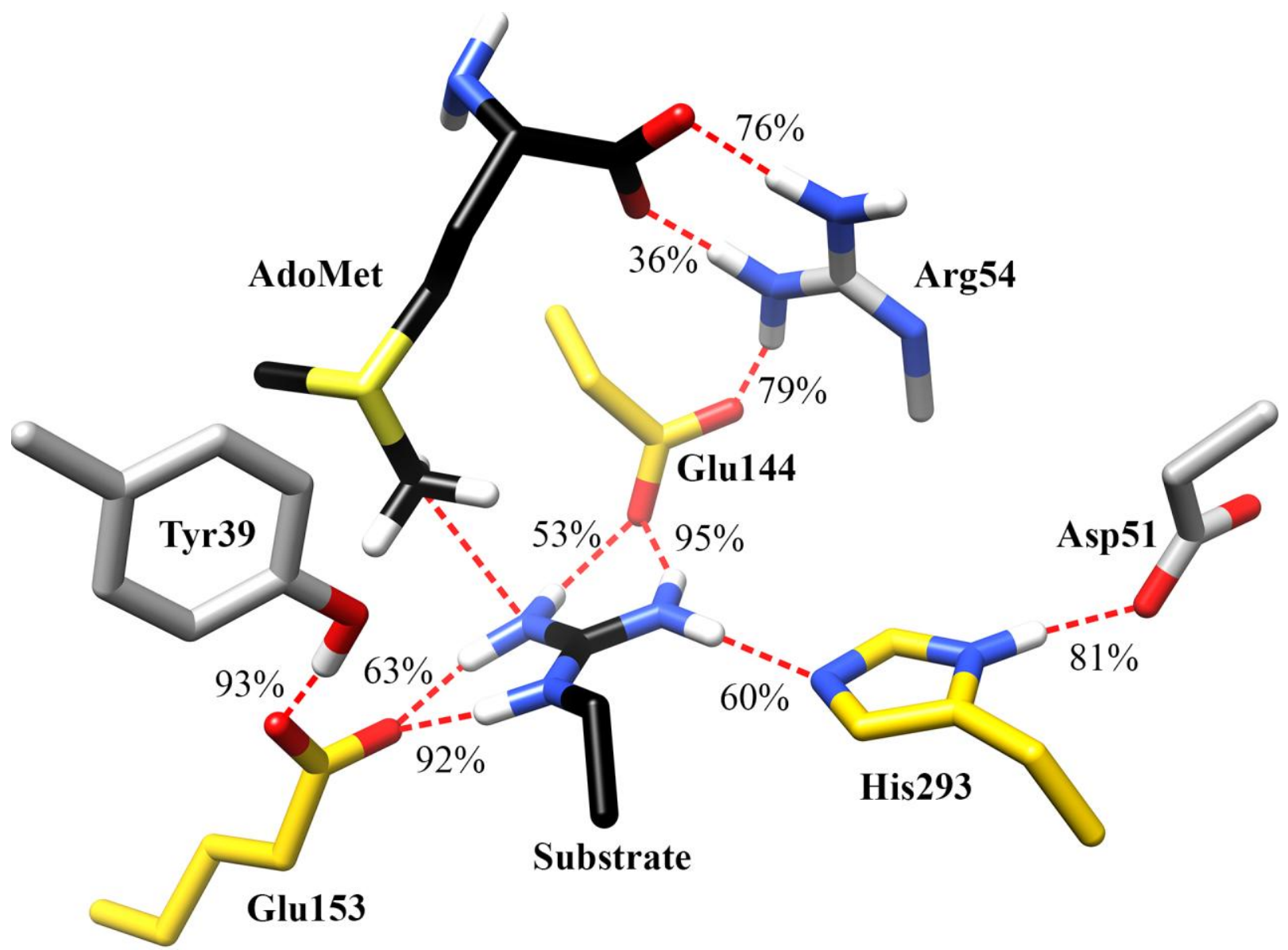

Figure 7. Percent occupancy of hydrogen bonding involving the GGRGG substrate, AdoMet, and nearby active site residues over the entire MD simulation for the WT PRMT1 enzyme.

Upon formation of MMA, there is the potential for the methyl group to be oriented in four different positions within the active site (Figure 8). Molecular mechanics/PoissonBoltzmann surface area (MM/PBSA) calculations ${ }^{49}$ were performed to compare the binding free energy $\left(\Delta G_{\text {bind }}\right)$ differences between the four MMA conformations in the PRMT1 active site (Table 2). The MM/PBSA calculations predicted higher $\Delta G_{\text {bind }}$ values for the subsequent MMA products compared to the unmethylated GGRGG substrate in the active site regardless of 
orientation. Among the four potential MMA conformations, when the methyl group is on the $\mathrm{N}_{\eta 1}$ nitrogen and facing "upwards" its $\Delta \Delta G_{\text {bind }}$ is favored by $5-27 \mathrm{kcal} / \mathrm{mol}$ compared to the other three $\mathrm{N}_{\eta 1^{-}}$and $\mathrm{N}_{\eta 2}$-methyl orientations (Figure 8 and Table 2). In this MMA- $\mathrm{N}_{\eta 1^{-}}$ "up"arrangement, the guandinio methyl group on $\mathrm{N}_{\eta 1}$ points towards the loop adjacent to the binding cavity of the substrate and the hydrogen bonds between Glu153, Glu144, and His293 are preserved compared to WT-Arg; the Trp145 and Met146 residues on that loop face away from the active site avoiding unfavorable steric hindrance (Supplemental Figure S6).

Table 2. Computed Binding Free Energies, $\Delta G_{\text {bind, }}$, $(\mathrm{kcal} / \mathrm{mol})$ using MM/PBSA for methylated and unmethylated GGRGG substrates in the PRMT1 active site. ${ }^{\text {a }}$

\begin{tabular}{|c|c|}
\hline Complex & $\Delta G_{\text {bind }}$ \\
\hline WT-Arg & -20.1 \\
\hline 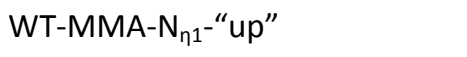 & -11.1 \\
\hline WT-MMA-N ${ }_{n 1}$-"down" & 15.9 \\
\hline WT-MMA-N ${ }_{\text {n2-“up” }}$ & -0.3 \\
\hline WT-MMA-N ${ }_{n 2}$-"down” & -6.1 \\
\hline M48F-Arg & -18.8 \\
\hline M48F-MMA-N ${ }_{n 1}$ “up” & 3.6 \\
\hline M48F-MMA-N ${ }_{n 2}-"$ down" & -2.8 \\
\hline H293S-M48F-Arg & -24.1 \\
\hline H293S-M48F-MMA-N ${ }_{n 1}{ }^{-“ u p ” ~}$ & 4.1 \\
\hline H293S-M48F-MMA-N ${ }_{n 2}-$ "down” & 2.9 \\
\hline
\end{tabular}

"For definition of "up" and "down" see Figure 8. 


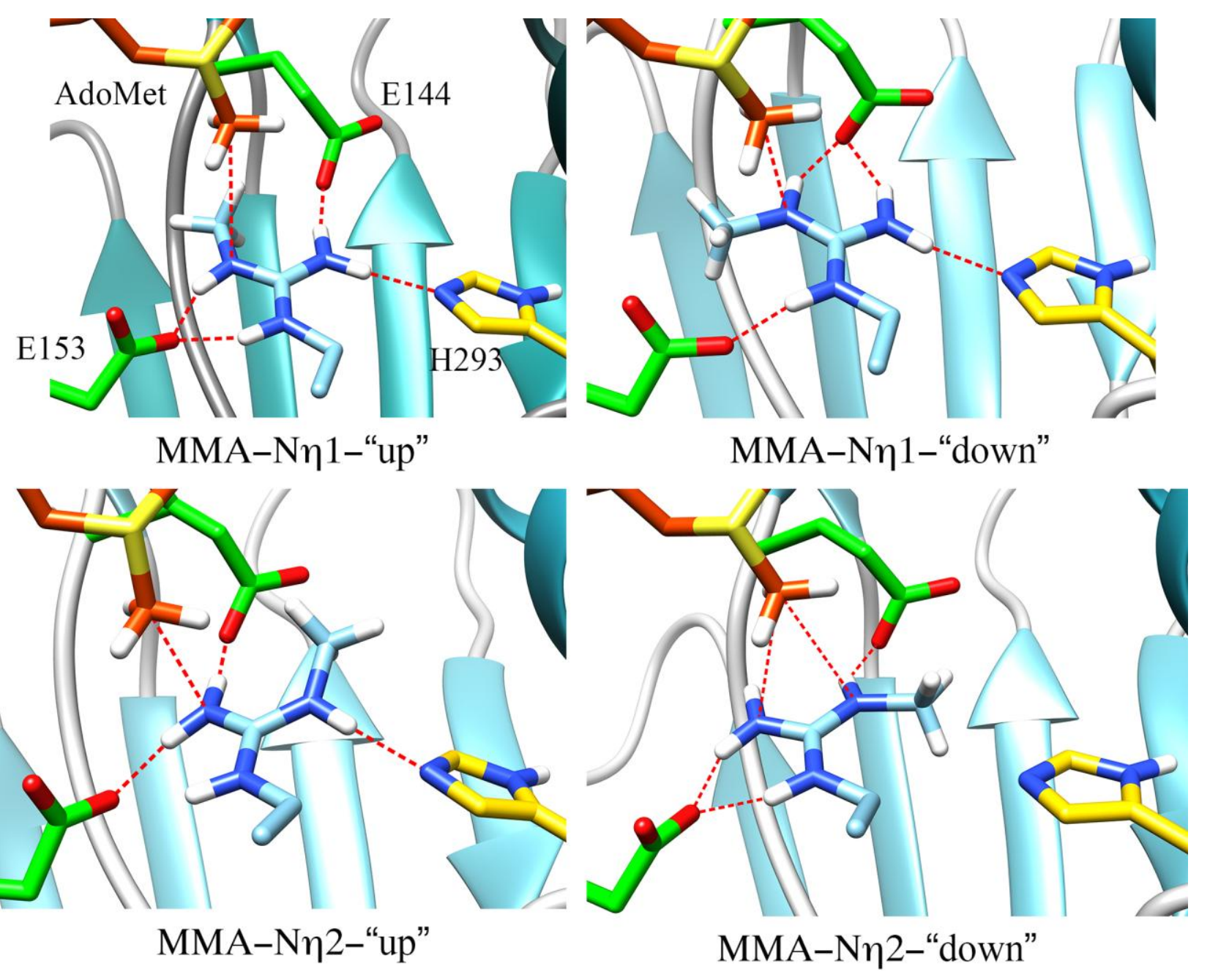

Figure 8. The methyl group oriented in four different positions for MMA within the WT PRMT1 active site.

For all subsequent mutant PRMT1 MD simulations, the MMA-N ${ }_{\eta 1}$ “"up” and MMA- $\mathrm{N}_{\eta 2}$ "down" orientations were performed exclusively due to the more favorable energetics computed in the WT system (Table 1) and from here on will be simply called MMA-N $\mathrm{N}_{11}$ and MMA-N ${ }_{\eta 2}$, respectively. The probability distributions found an overall reduction in the formation of NACs, i.e., much smaller peaks centered around $3.1-3.2 \AA$, for the WT-MMA-N $\mathrm{N}_{11}$ configuration compared to the WT-Arg plot (Figure 4). The hydrogen bonding percent occupancy between 
Glu144 and MMA- $\mathrm{N}_{\eta 1}$ is considerably lower at $55.1 \%$ compared to $94.5 \%$ for the WT-Arg system (Figures 7 and 9 and Table 1). Consistent with PRMT1's ability to form ADMA as the exclusive second turnover product, the probability distributions for WT-MMA-N $\mathrm{N}_{11}$ did predict a more facile ability to form NACs favoring ADMA formation with a second peak centered around $5.2 \AA$, compared to $6.4 \AA$ for SDMA. The WT-MMA-N $\mathrm{N}_{2}$ orientation yielded broader and smaller probability peaks indicative of a substantial reduction in hydrogen bond stabilization of the substrate within the active site, particularly during SDMA formation. Accordingly, monitoring the $300 \mathrm{~ns}$ trajectory for the WT-MMA- $\mathrm{N}_{\eta 2}$ simulation finds that the guanidino moiety veers away from the $\mathrm{CH}_{3}$ group on AdoMet over time to much longer distances of $10-$ $15 \AA$ with the first 2 helices of the N-terminal eventually opening up the active site.

The simulations emphasize that specific intermolecular interactions occurring between Arg54, Glu144, Glu153, His293, the GGRGG substrate, and the AdoMet cofactor are particularly important in stabilizing the ternary complex for the arginine methylation reaction. For example, Arg54 makes key hydrogen bonds that fix AdoMet and Glu144 into the appropriate position for the $S_{N} 2$ mechanism (Scheme 1). A similar interaction involving Tyr39 and Glu153 is ever-present with hydrogen bonding occupancies above $90 \%$, particularly in the WT systems (Figure 7 and Table 1). The importance of this interaction was experimentally highlighted by the enzyme's pH profile where the protonation and deprotonation of Tyr39 and Glu153 must be achieved for optimal PRMT1 activity. ${ }^{29}$

Favorable intermolecular interactions between AdoMet and nearby active site residues, e.g., Arg54, Thr81, and Glu100, were monitored for the WT and mutant PRMT1 enzymes (Figure 9). The carboxylate group of Glu100 was found to anchor AdoMet in the active site by forming hydrogen bonds with the hydroxyl groups of the ribose moiety with a very high 


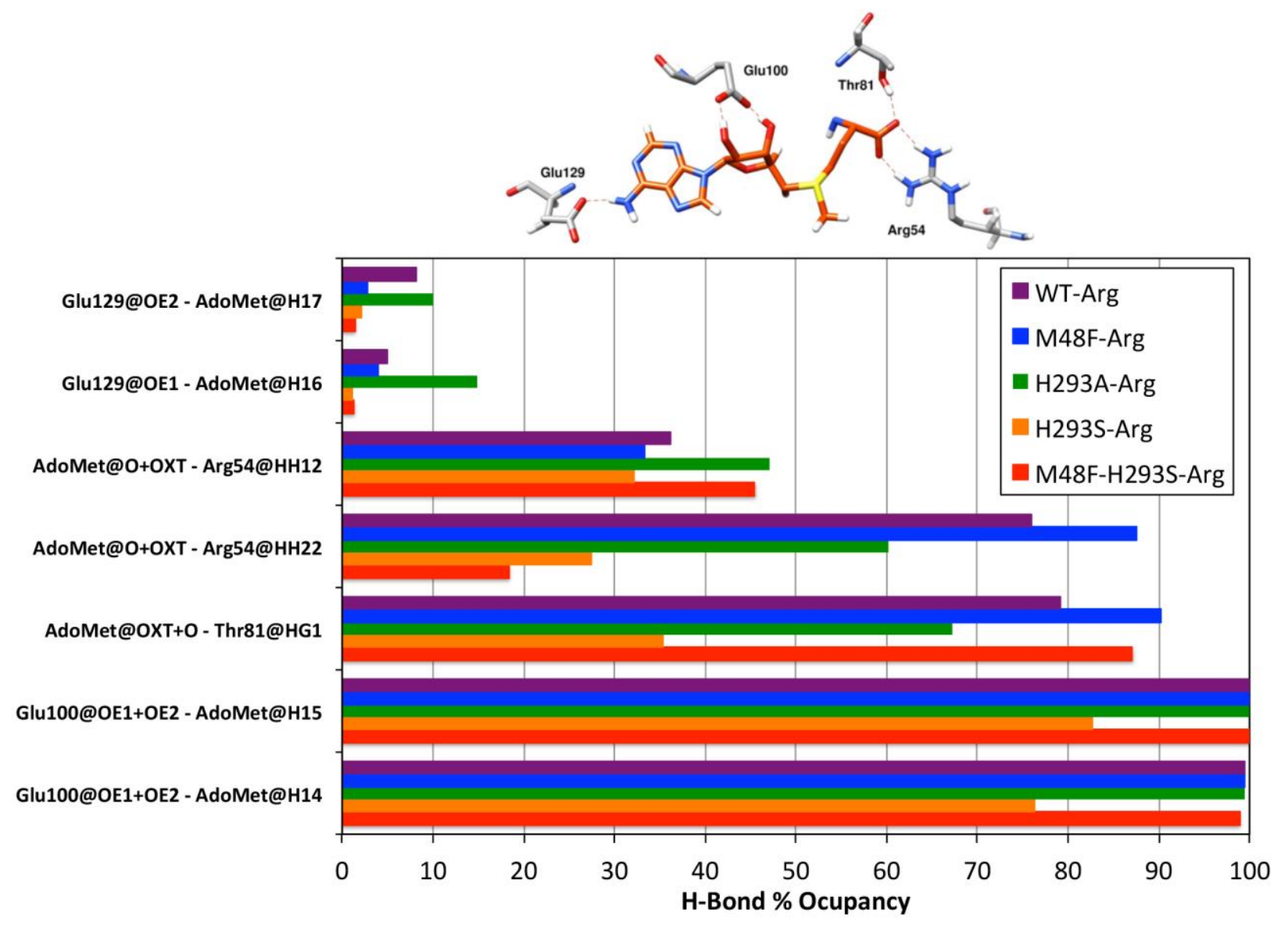

Figure 9. Percent occupancy of hydrogen bonding (acceptor-donor) involving AdoMet and active site residues over the entire simulation for the WT, M48F, H293A, H293S, and H293SM48F PRMT1 systems.

occupancy of 99-100\% in all the WT and mutant PRMT1 simulations (Supplemental Table S1). In addition, the methionine moiety is held in position by hydrogen bonds with Thr81 and Arg54 for a significant portion of the simulation. However, despite a potential interaction distance between the adenosyl group on AdoMet and Glu129 conducive to favorable electrostatic interactions, very little appreciable hydrogen bonding occurred with less than $10 \%$ occupancy computed over the course of each trajectory. Other important intermolecular interactions for AdoMet in the active site were primarily hydrophobic in nature, as residues Met155 and Met48 
have been shown to be particularly important for AdoMet binding, ${ }^{25}$ catalysis, ${ }^{34}$ and product specificity. $^{33,34}$

3.2 H293S/A-PRMT1. His293 has been hypothesized to form a salt bridge with a conserved residue Asp51 that plays a critical role in forming the two-helix boundary that separates the AdoMet and peptide binding portions of the active site (Figure 10). ${ }^{29}$ Accordingly, this salt bridge interaction was ubiquitous in the WT-Arg and M48F-Arg PRMT1 simulations with hydrogen bonding percent occupancies of $81.3 \%$ and $87.1 \%$, respectively, over each $300 \mathrm{~ns}$ trajectory (Table 1). Despite the $6 \AA$ distance between His293 and the substrate guanidinium reported in the AdoHcy-bound PRMT1 crystal structure, ${ }^{25}$ the current MD simulations found significant movement occurring within the active site with AdoMet present. The result is a favorable interacting distance between the residue-substrate pair with hydrogen bonding percent occupancies of $60.4 \%$ and $61.0 \%$ for the WT-Arg and M48F-Arg systems, respectively (Figure 7). The methylation of the GGRGG substrate reduced the hydrogen bonding occupancy between His293 and the substrate to $49.0 \%$ and $25.2 \%$ for the WT-MMA-N $\mathrm{N}_{\eta 1}$ and M48F-MMA-N $\mathrm{N}_{\eta 1}$ systems, respectively, whereas the His293-Asp51 salt bridge retained substantial values of $87.1 \%$ and $83.5 \%$ (Table 1). No hydrogen bonding is possible between His293 and the WT-MMA-N $\mathrm{N}_{\eta 2}$ substrate as the methyl group on $\mathrm{N}_{\eta^{2}}$ occupies the potential hydrogen bonding position (Figure 8). 


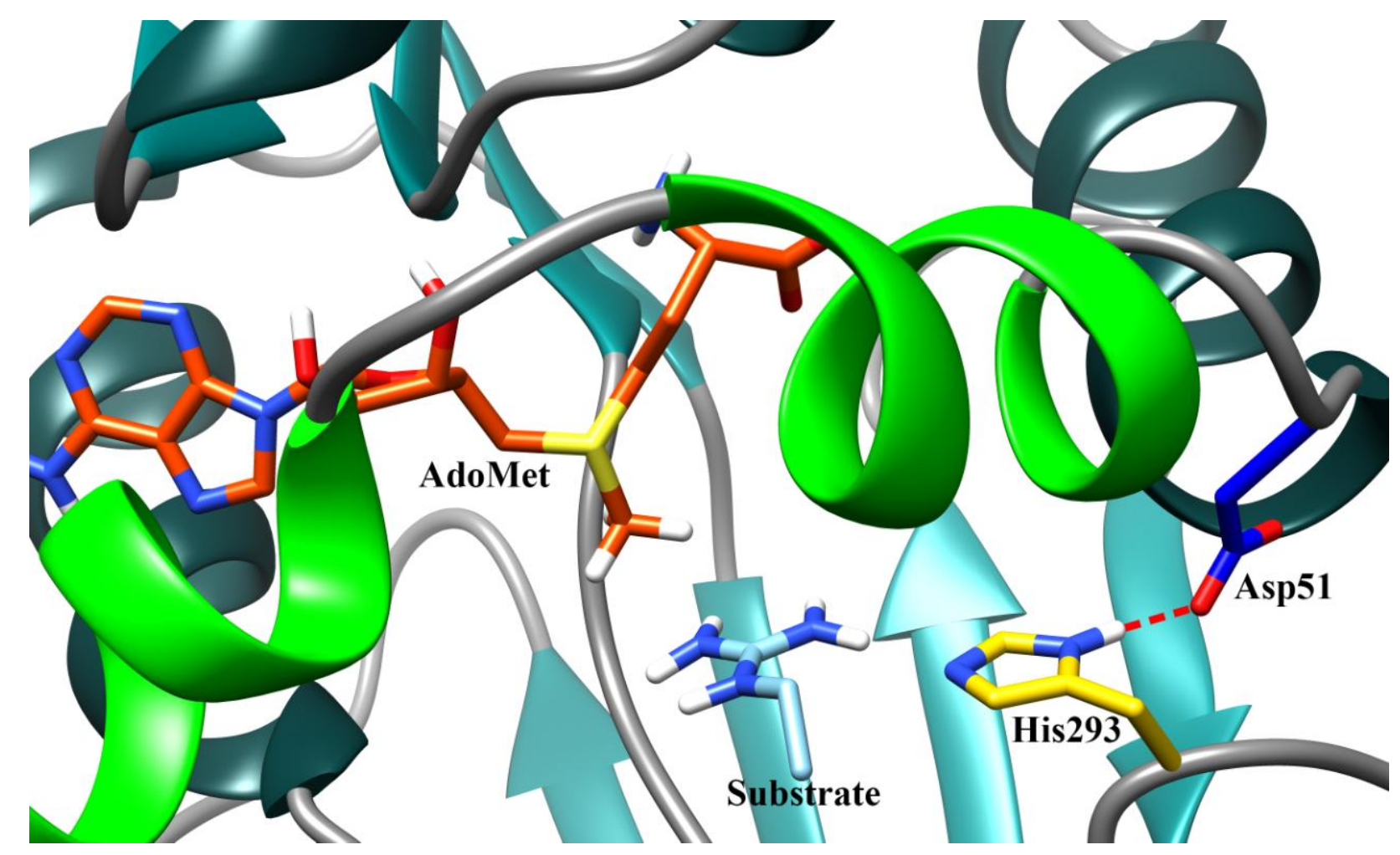

Figure 10. Salt bridge between His293 and Asp51 in the PRMT1 active site. The N-terminal helix is shown in green.

The H293A-PRMT1 mutant should impair both the potential for salt bridge formation with Asp51 and hydrogen bonding with the GGRGG substrate. Experimental mutagenesis studies of H293A-PRMT1 reported decreased $k_{\text {cat }} / K_{\mathrm{m}}$ values for an AcH4-21 peptide substrate and AdoMet by 256- and 50-fold, respectively, driven predominantly by $k_{\text {cat. }}{ }^{29}$ The decreased activity is attributed to a disrupted binding pocket as crucial residues present on the helix, e.g., Tyr39, His45, Met48, and Arg54, play large roles in AdoMet and substrate stabilization. Simulations were performed on the H293A-PRMT1 system and the integrity of the active site remained primarily intact throughout the $300 \mathrm{~ns}$ simulation. H293A-Arg hydrogen bonding percent occupancies between the aforementioned active site residues and AdoMet were 
comparable to WT-Arg, but the closest interactions between GGRGG and Glu144 and Glu153 were reduced to $74.5 \%$ and $47 \%$ compared to $92-94 \%$ in the WT (Supporting Information Table S2). A longer MD simulation or enhanced sampling methods may be required to find any larger structural changes. However, perhaps His293's involvement in modulating the nucleophilicity of the arginine substrate, or even a possible role for His293 as the general base, should not be undervalued.

In the solved crystal structure of PRMT5, a Type II enzyme capable of producing MMA and SDMA products exclusively, a serine residue exists in the corresponding location of His293 in rat PRMT1. ${ }^{54}$ A H293S-PRMT1 mutant should be capable of favorable intermolecular interactions with Asp51 and/or with the arginine on the substrate. However, the calculated hydrogen bonding between Ser293 and the GGRGG substrate was dramatically lower with percent occupancies of $4.7 \%$, and $0.4 \%$ for the H293S-Arg and H293S-MMA-N $\mathrm{N}_{\eta 1}$ systems, respectively, over the course of each 300 ns trajectory (Supplemental Table S2). The interaction between Ser293 and Asp51 also had reduced hydrogen bonding occupancies for H293S-Arg and H293S-MMA-N $\mathrm{N}_{11}$ with values of $19.5 \%$ and $24.7 \%$ compared to the higher $\sim 81-87 \%$ for the WT and M48F-PRMT1 systems (Table 1 and Supplemental Table S2). The H293S-PRMT1 MMA$\mathrm{N}_{\eta 2}$ complex was found to be unstable as the substrate exited the active site after 130 ns for two different MD trajectories.

Computed average $d+0.5(\cos \theta)$ distances for the H293S-PRMT1 enzyme bound with the Arg and MMA-N $\mathrm{N}_{\eta 1}$ substrates found reduced probabilities of forming an NAC to yield the MMA, ADMA, and SDMA products, with peaks less than half of the height of the WT-PRMT1 system (Supplemental Figure S7). The probability peaks also shifted to larger distances with H293S-Arg average values of 5.6 and $4.6 \AA$ to form the MMA-N $\mathrm{N}_{\eta 1}$ and MMA-N $\mathrm{N}_{\eta 2}$ substrate 
products. From the simulations, it is not expected that product specificity would be altered relative to WT-PRMT1, but that the enzyme's activity should be impaired. To test the prediction, the H293S-PRMT1 mutant was experimentally generated and product formation was analyzed using (RP)-HPLC with trace amounts of tritium-labeled AdoMet (Figure 11). The (RP)-HPLC analysis method has been shown to be a reliable tool for identifying methylarginine products. ${ }^{34}$ Separation of standard MMA, ADMA, and SDMA was evident. Confirming simulation results, the H293S-PRMT1 mutant exclusively formed the MMA and ADMA products associated with Type I PRMTs, but in reduced amounts as compared to WT-PRMT1 (Figure 11 A and B).
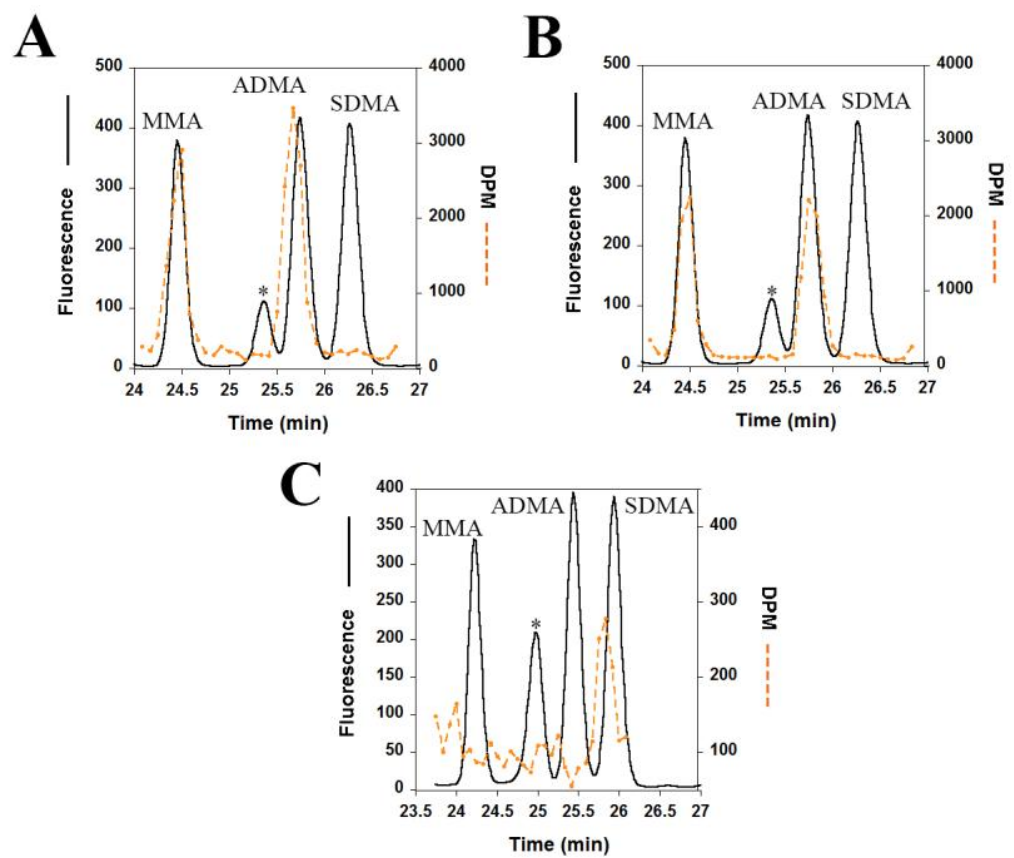

Figure 11. Analysis of His-ratPRMT1 variant-catalyzed methylation products by reverse phase HPLC. Products from reactions catalyzed by (A) wild-type, (B) H293S, and (C) H293S-M48FPRMT1 are shown. $\left[{ }^{3} \mathrm{H}\right] \mathrm{S}$-adenosyl methionine was used as a tracer to verify the presence of the methylated arginine product in each reaction (orange trace). Standards for the three arginine 
products: MMA, ADMA, and SDMA (solid black line). The peak labeled with an asterisk is a contaminant peak.

3.3 M48F-PRMT1 and H293S-M48F-PRMT1. Our previous study reported that mutation of Met48 to phenylalanine in PRMT1 enabled the mutated enzyme to generate SDMA as a new product. ${ }^{34}$ However, limited SDMA formation was observed, i.e., relative abundance of $0.10 \%$. Quantum mechanical calculations predicted a larger energetic cost for the formation of SDMA, which may explain the impaired formation, but those gas-phase calculations lacked the full enzyme environment. Probability distributions were again computed here for each $300 \mathrm{~ns}$ MD trajectory of the M48F-PRMT1 with the bound GGRGG substrate and AdoMet. The M48FArg probability distribution peaks for forming a NAC to yield the MMA product were comparable to that of WT-Arg PRMT1 (Figures 4 and 12). The average $d+0.5(\cos \theta)$ distances were computed to be 3.3 and $3.9 \AA$ between the reactive AdoMet methyl-C atom and the $\mathrm{N}_{\eta 1}$ and $\mathrm{N}_{\eta 2}$ atoms, respectively, indicative of a MMA- $\mathrm{N}_{\eta 1}$ product formation preference for M48F-Arg; on the contrary, probability distributions for WT-PRMT1 found very little preferential difference between $N_{\eta 1}$ and $N_{\eta 2}$ MMA attack formation (Figure 5). Visual inspection of a crystal structure overlay between the Type II PRMT5 enzyme and a manually inserted MMA- $\mathrm{N}_{\eta_{2}}$ substrate suggests that the given orientation for the methylated arginine substrate would also result in unfavorable steric collisions with Phe379. ${ }^{34}$ Yet, experimental mutagenesis of M48 into smaller hydrophobic residues in PRMT1, such as alanine or proline, did not form SDMA product, but reduced catalytic efficiencies by 338 - and 70 -fold, respectively. ${ }^{33}$ This may imply that different binding modes for MMA in PRMT1 and PRMT5 could in part govern product specificity. 
The predicted second turnover reaction for M48F-PRMT1 leading to either ADMA or SDMA had significantly shorter $d+0.5(\cos \theta)$ average probability distances compared to WT PRMT1. For example, M48F-MMA- $\mathrm{N}_{\eta 1}$ gave an average NAC distance of $3.9 \AA$ between the methyl-C atom and the $\mathrm{N}_{\eta 1}$ atom leading to ADMA and 3.5 $\AA$ for the $\mathrm{N}_{\eta 2}$ atom leading to SDMA (Figure 5). Both distances are much more favorable than the WT PRMT1 average distances of 4.9 and $5.8 \AA$ for ADMA and SDMA products, respectively. The calculations predicted M48FPRMT1 could generate both second turnover products (Figure 12), which is consistent with experimental findings. ${ }^{34}$ However, the M48F-MMA- $\mathrm{N}_{\eta^{2}}$ orientation gave much larger average $d$ $+0.5(\cos \theta)$ distances of 5.8 and $6.3 \AA$ for the $\mathrm{N}_{\eta_{1}}$ and $\mathrm{N}_{\eta^{2}}$ atoms, respectively, signifying poorer binding in that orientation. These factors could be indicative of the reduced efficiency found in the M48F-PRMT1 mutant. ${ }^{34}$ Of course, it should be noted that positions alone could not capture all factors that determine reactivity. Other considerations include a potentially favorable cation- $\pi$ interaction between the positively charged guanidino substrate and Phe48's aromatic ring. Energetically significant cation- $\pi$ interactions are ubiquitous in proteins and are regarded as an important noncovalent binding interaction relevant to structural biology. ${ }^{55}$ 


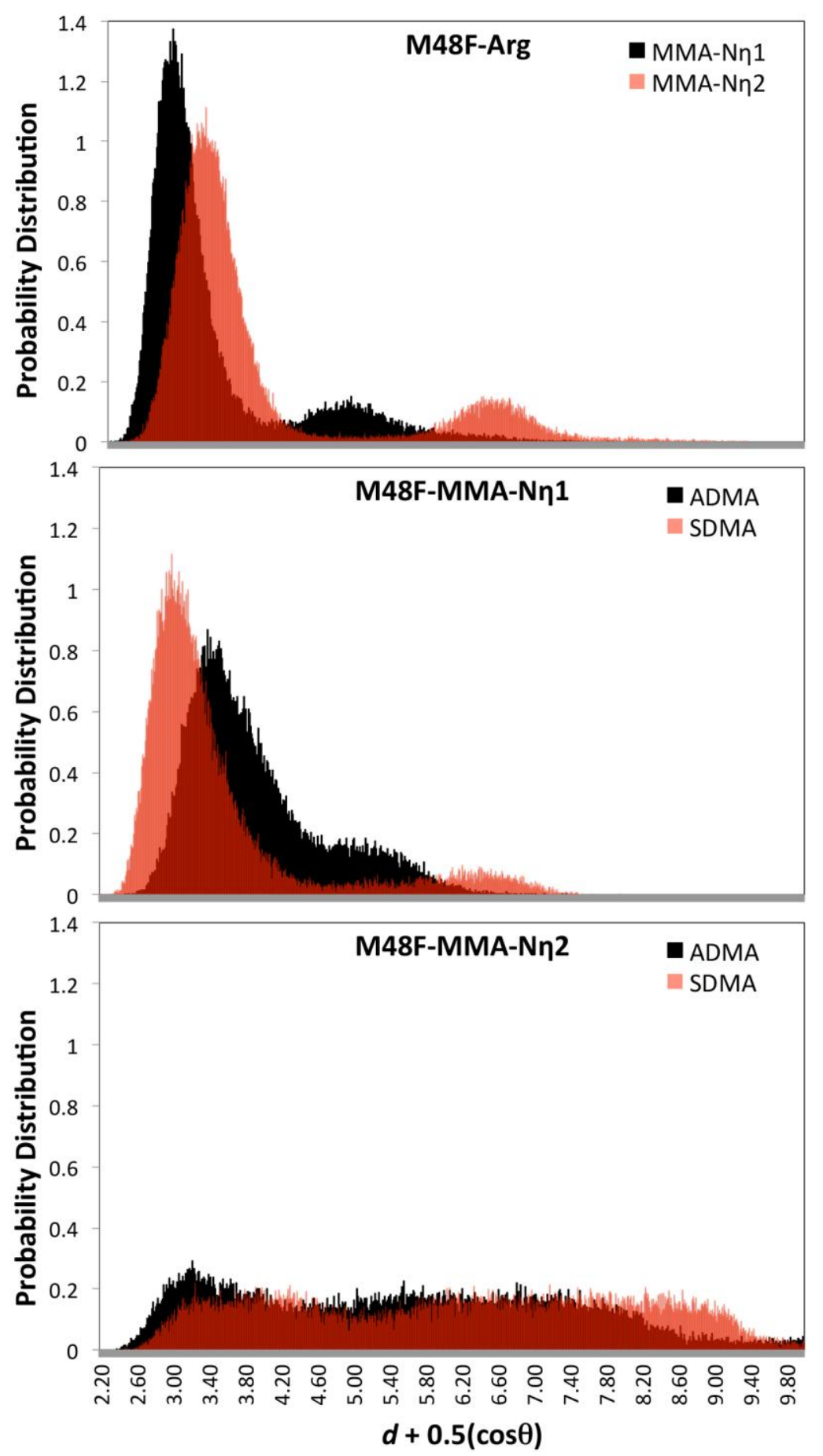

Figure 12. Probability of sampling near-attack conformers in the M48F-PRMT1 complex that leads to the formation of MMA, ADMA, or SDMA products. The preferred orientations by the substrate over time were evaluated by comparing the distributions of $d+0.5(\cos \theta)$ for all 
trajectories, where $d$ is the attack distance between the nitrogen atom in arginine $\left(\mathrm{N}_{\eta 1}\right.$ and $\left.\mathrm{N}_{\eta 2}\right)$ and the methyl carbon in AdoMet, and $\theta$ is the respective attack angle $\left(\mathrm{N}^{\cdots} \mathrm{CH}_{3} \cdots \mathrm{S}\right)$.

Additional structural features beyond the active site Phe/Met switch may play a prominent role in controlling product specificity, as the Type II enzyme PRMT9 possesses a methionine and not a phenylalanine at the corresponding position. ${ }^{1}$ The presence of the Phe 48 in the M48F-PRMT1 simulations halved the hydrogen bonding percent occupancy between GGRGG and His293 from 49.0\% in WT-MMA-N $\mathrm{N}_{\eta 1}$ to $25.2 \%$ for M48F-MMA-N $\mathrm{N}_{11}$ (Table 1) potentially indicating a reduced role for His 293 in the mutated enzyme. Intriguingly, both Type II enzymes PRMT5 and PRMT9 lack the histidine found at position 293 in PRMT1 and instead contain a corresponding serine and cysteine residue. It has been speculated that the smaller side chains may better accommodate the SDMA product in the active site. ${ }^{1}$ Accordingly, a H293SM48F-PRMT1 double mutant should increase active site volume to better resemble that of the Type II PRMT5 pocket.

Simulations were carried out for the H293S-M48F-Arg PRMT1 double mutant and average $d+0.5(\cos \theta)$ distances were computed to be 3.2 and $5.5 \AA$ between the reactive AdoMet methyl-C atom and the $\mathrm{N}_{\eta 1}$ and $\mathrm{N}_{\eta 2}$ atoms, respectively (Figure 5). The probability peaks for the formation of MMA were broad and shifted to larger distances, which is indicative of poorer MMA formation relative to WT, regardless of $\mathrm{N}_{\eta 1}$ or $\mathrm{N}_{\eta 2}$ orientation (Figure 13). However, after MMA creation, the H293S-M48F-MMA- $\mathrm{N}_{\eta_{2}}$ binding orientation was able to favorably accommodate the substrate within the active site, which is considerably different from the WT and other mutant simulations where poor sterics within the MMA- $\mathrm{N}_{\eta 2}$ pocket forced the substrate to exit the enzyme over the course of the trajectory. For example, the computed probability peaks 
for the MMA- $\mathrm{N}_{\mathrm{\eta} 2}$ orientations in WT and M48F PRMT1 systems yielded very small and broad peaks for NAC formation over a large distance range of 2.5 to $9.5 \AA$, which is indicative of a low chance of productive product formation (Figures 4 and 12). In stark contrast, the double mutant H293S-M48F-MMA-N $\mathrm{N}_{12}$ system gave narrower peaks with average $d+0.5(\cos \theta)$ distances of $3.2 \AA$ leading to more favorable SDMA formation compared to $4.0 \AA$ for the ADMA product (Figures 5 and 13). The H293S-M48F-MMA- $\mathrm{N}_{\eta 1}$ system also has narrow peaks, but more similar average $d+0.5(\cos \theta)$ distance values of 3.7 and $3.8 \AA$ when leading to ADMA and SDMA product, respectively. The simulations indicate that the H293S-M48F PRMT1 double mutant should produce both second turnover products, but favor SDMA formation. The H293S-M48FPRMT1 mutant was experimentally generated and product formation analyzed using (RP)HPLC. Accordingly, very small amounts of radioactivity eluted at the same retention time as MMA and ADMA, but notably, a significant amount of SDMA was formed (Figure 11). This double mutant PRMT1 represents the first time that a Type I PRMT can be tuned to generate more SDMA than its natural WT products MMA and ADMA. 


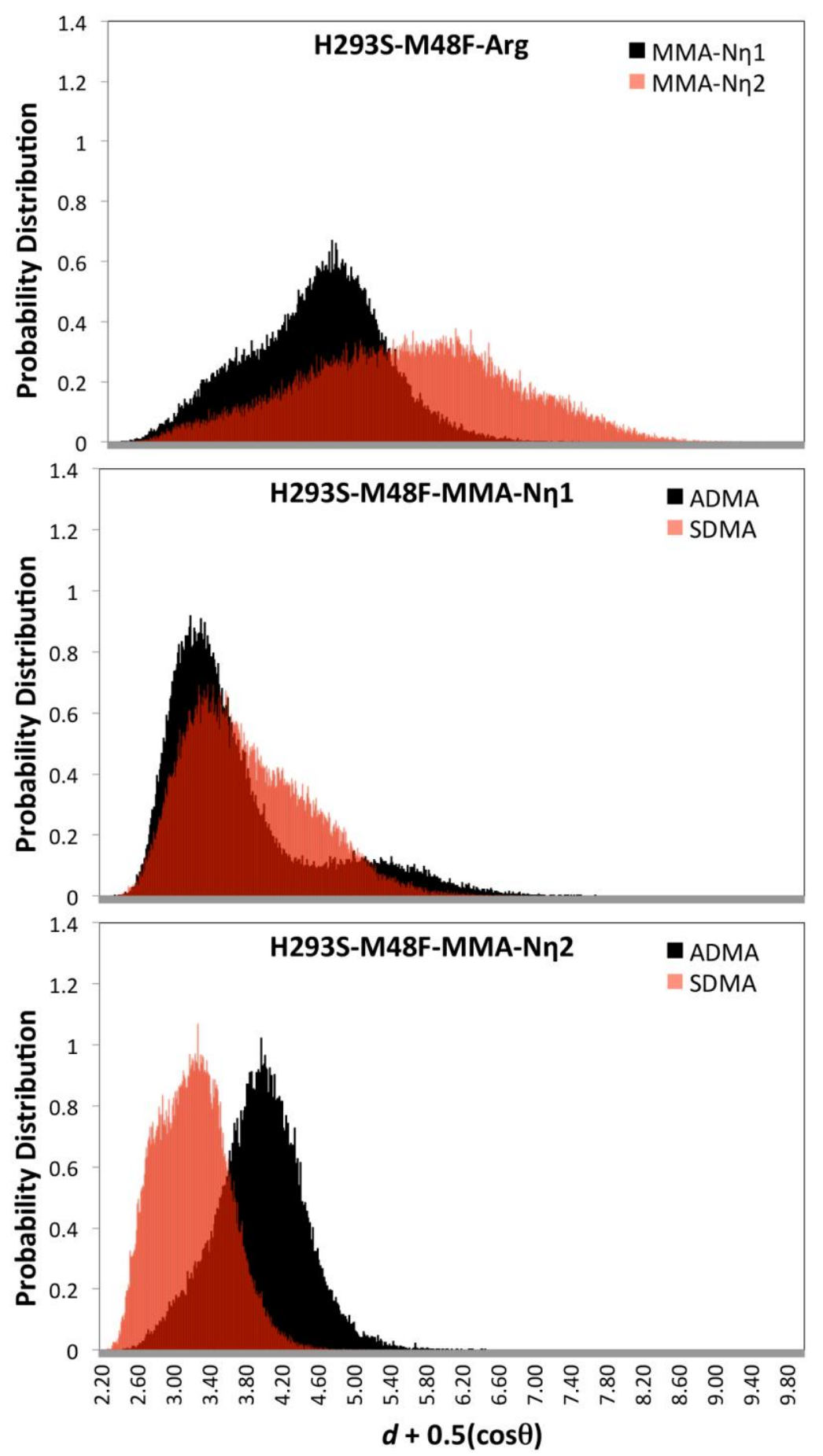

Figure 13. Probability of sampling near-attack conformers in the H239S-M48F-PRMT1 complex that leads to the formation of MMA, ADMA, or SDMA products. The preferred orientations by the substrate over time were evaluated by comparing the distributions of $d+$ $0.5(\cos \theta)$ for all trajectories, where $d$ is the attack distance between the nitrogen atom in arginine 
$\left(\mathrm{N}_{\eta 1}\right.$ and $\left.\mathrm{N}_{\eta 2}\right)$ and the methyl carbon in AdoMet, and $\theta$ is the respective attack angle $\left(\mathrm{N} \cdots \mathrm{CH}_{3} \cdots \mathrm{S}\right)$.

\section{Conclusions}

Molecular dynamics simulations were conducted for the WT, M48F, H293A, H293S, and H293S-M48F PRMT1 enzyme systems bound with AdoMet and either a Gly-Gly-Arg-Gly-Gly (GGRGG) substrate or the monomethylated GGRGG substrate. The simulations emphasize that specific intermolecular interactions occurring between active site residues Glu144, Glu153, Arg54, Thr81, and Glu100, and the GGRGG substrate and AdoMet cofactor are important in stabilizing the ternary complex for the arginine methylation reaction. However, two residues, Met48 and His293, were found to be particularly significant in controlling the product specificity in PRMT1. Calculating the probability of sampling near-attack conformers led to the prediction that the H239S-M48F-PRMT1 mutant should produce SDMA as a major product, as opposed to the MMA and ADMA products exclusively made by Type I PRMT enzymes. Experimental sitedirected mutagenesis and analysis of the resultant products of the M48F, H293S, and H293SM48F mutants were carried out to test the hypothesis. Product formation analyzed using (RP)HPLC determined that the M48F mutation enabled PRMT1 to produce a limited amount of SDMA product in addition to the expected MMA and ADMA products associated with Type I PRMT enzymes. The H293S PRMT1 mutant only produced the expected MMA and ADMA with reduced efficiency. However, the combination of both mutations, i.e., H293S-M48F PRMT1, generated a substantial amount of SDMA product, with very little MMA and ADMA produced, as predicted from the simulations. Accordingly, it was previously reported that the Type II F379M-PRMT5 mutant (where Ser669 is the equivalent residue of His293 in PRMT1) 
also produced both SDMA and ADMA second turnover products and displayed appreciably greater methylation activity than WT-PRMT5. ${ }^{54}$ This highlights that the corresponding M48/F379 and H293/S669 positions in the PRMT1/PRMT5 active sites are largely responsible for controlling product specificity. The hydrogen bonding interaction between His293 and the substrate indicates that it could be involved in determining substrate orientation, previously dismissed due to a $6 \AA$ distance in the reported crystal structure. ${ }^{25}$ The calculations suggest that the combination of Met48 and His293 may enable PRMT1 to yield ADMA exclusively by precluding MMA from binding with the methyl group in the $\mathrm{N}_{\eta 2}$ position, which is more favorable for SDMA formation in the H293S-M48F PRMT1 mutant.

Acknowledgement. Gratitude is expressed to the National Science Foundation (CHE-1626860, CHE-1412358 to O.A. and CHE-1412405 to J.M.H.), Auburn University, and the Alabama Supercomputer Center for support of this research.

\section{References}

(1) Fuhrmann, J.; Clancy, K. W.; Thompson, P. R. Chemical biology of protein arginine modifications in epigenetic regulation. Chem. Rev. 2015, 115, 5413-5461.

(2) Kleinschmidt, M. A.; Graaf, P. d.; Teeffelen, H. A. A. M. v.; Timmers, H. T. M. Cell cycle regulation by the PRMT6 arginine methyltransferase through repression of cyclin-dependent kinase inhibitors. PLOS One 2012, 7, e41446.

(3) Blackwell, E.; Ceman, S. Arginine methylation of RNA-binding proteins regulates cell function and differentiation. Mol. Reprod. Dev. 2012, 79, 163-175.

(4) Batut, J.; Duboé, C.; Vandel, L. The methyltransferases PRMT4/CARM1 and PRMT5 control differentially myogenesis in zebrafish. PLOS One 2011, 6, e25427. 
(5) Chen, C.; Nott, T. J.; Jin, J.; Pawson, T. Deciphering arginine methylation: Tudor tells the tale. Nat. Rev. Mol. Cell. Bio. 2011, 12, 629-642.

(6) Lee, Y.-H.; Stallcup, M. R. Roles of protein arginine methylation in DNA damage signaling pathways is CARM1 a life-or-death decision point? Cell Cycle 2011, 10, 1343-1344.

(7) Bedford, M. T.; Clarke, S. G. Protein arginine methylation in mammals: Who, what, and why. Mol. Cell 2009, 33, 1-13.

(8) Lee, Y.-H.; Stallcup, M. R. Minireview: Protein arginine methylation of nonhistone proteins in transcriptional regulation. Mol. Endocrinol. 2009, 23, 425-433.

(9) Wolf, S. S. The protein arginine methyltransferase family: An update about function, new perspectives and the physiological role in humans. Cell. Mol. Life Sci. 2009, 66, 2109-2121.

(10) Schubert, H. L.; Blumenthal, R. M.; Cheng, X. Many paths to methyltransfer: A chronicle of convergence. Trends Biochem Sci 2003, 28, 329-335.

(11) Bachand, F. Protein arginine methyltransferases: From unicellular eukaryotes to humans. Eukaryot Cell 2007, 6, 889-898.

(12) Leiper, J.; Nandi, M.; Torondel, B.; Murray-Rust, J.; Malaki, M.; O'Hara, B.; Rossiter, S.; Anthony, S.; Madhani, M.; Selwood, D.; Smith, C.; Wojciak-Stothard, B.; Rudiger, A.; Stidwill, R.; McDonald, N. Q.; Vallance, P. Disruption of methylarginine metabolism impairs vascular homeostasis. Nature medicine 2007, 13, 198-203.

(13) Chen, D.; Ma, H.; Hong, H.; Koh, S. S.; Huang, S. M.; Schurter, B. T.; Aswad, D. W.; Stallcup, M. R. Regulation of transcription by a protein methyltransferase. Science 1999, 284, 2174-2177.

(14) Hong, H.; Kao, C.; Jeng, M. H.; Eble, J. N.; Koch, M. O.; Gardner, T. A.; Zhang, S.; Li, L.; Pan, C. X.; Hu, Z.; MacLennan, G. T.; Cheng, L. Aberrant expression of CARM1, a transcriptional coactivator of androgen receptor, in the development of prostate carcinoma and androgen-independent status. Cancer 2004, 101, 83-89.

(15) Majumder, S.; Liu, Y.; Ford, O. H., 3rd; Mohler, J. L.; Whang, Y. E. Involvement of arginine methyltransferase carm1 in androgen receptor function and prostate cancer cell viability. The Prostate 2006, 66, 1292-1301.

(16) Tang, J.; Frankel, A.; Cook, R. J.; Kim, S.; Paik, W. K.; Williams, K. R.; Clarke, S.; Herschman, H. R. PRMT1 is the predominant type I protein arginine methyltransferase in mammalian cells. J. Biol. Chem. 2000, 275, 7723-7730. 
(17) Lee, W.-C.; Lin, W.-L.; Matsui, T.; Chen, E. S.-W.; Wei, T.-Y. W.; Lin, W.-H.; Hu, H.; Zheng, Y. G.; Tsai, M.-D.; Ho, M.-C. Protein arginine methyltransferase 8: Tetrameric structure and protein substrate specificity. Biochemistry 2015, 54, 7514-7523.

(18) Yang, Y.; Hadjikyriacou, A.; Xia, Z.; Gayatri, S.; Kim, D.; Zurita-Lopez, C.; Kelly, R.; Guo, A.; Li, W.; Clarke, S. G.; Bedford, M. T. PRMT9 is a type II methyltransferase that methylates the splicing factor sap145. Nat. Commun. 2015, 6, 6428.

(19) Feng, Y.; Maity, R.; Whitelegge, J. P.; Hadjikyriacou, A.; Lill, Z.; Zurita-Lopez, C.; AlHadid, Q.; Clark\|, A. T.; Bedford, M. T.; Masson, J.-Y.; Clarke, S. G. Mammalian protein arginine methyltransferase 7 (PRMT7) specifically targets rxr sites in lysine- and arginine-rich regions. J. Biol. Chem. 2013, 288, 37010-37025.

(20) Kirmizis, A.; Santos-Rosa, H.; Penkett, C. J.; Singer, M. A.; Green, R. D.; Kouzarides, T. Distinct transcriptional outputs associated with mono- and dimethylated histone h3 arginine 2. Nat. Struct. Mol. Biol. 2009, 16, 449-451.

(21) Zhao, Q.; Rank, G.; Tan, Y. T.; Li, H.; Moritz, R. L.; Simpson, R. J.; Cerruti, L.; Curtis, D. J.; Patel, D. J.; Allis, C. D.; Cunningham, J. M.; Jane, S. M. PRMT5-mediated methylation of histone h4r3 recruits dnmt3a, coupling histone and DNA methylation in gene silencing. Nat. Struct. Mol. Biol. 2009, 16, 304-311.

(22) Strahl, B. D.; Briggs, S. D.; Brame, C. J.; Caldwell, J. A.; Koh, S. S.; Ma, H.; Cook, R. G.; Shabanowitz, J.; Hunt, D. F.; Stallcup, M. R.; Allis, C. D. Methylation of histone h4 at arginine 3 occurs in vivo and is mediated by the nuclear receptor coactivator prmt1. Curr. Biol. 2001, 11, 996-1000.

(23) Wang, H.; Huang, Z.-Q.; Xia, L.; Feng, Q.; Erdjument-Bromage, H.; Strahl, B. D.; Briggs, S. D.; Allis, C. D.; Wong, J.; Tempst, P.; Zhang, Y. Methylation of histone h4 at arginine 3 facilitating transcriptional activation by nuclear hormone receptor. Science 2001, 293, 853-857.

(24) Morales, Y.; Cáceres, T.; May, K.; Hevel, J. M. Biochemistry and regulation of the protein arginine methyltransferases (PRMTs). Arch. Biochem. Biophys. 2016, 590, 138-152.

(25) Zhang, X.; Cheng, X. Structure of the predominant protein arginine methyltransferase prmt1 and analysis of its binding to substrate peptides. Structure 2003, 11, 509-520.

(26) Kölbel, K.; Ihling, C.; Bellmann-Sickert, K.; Neundorf, I.; Beck-Sickinger, A. G.; Sinz, A.; Kühn, U.; Wahle, E. Type I arginine methyltransferases PRMT1 and PRMT3 act distributively. J. Biol. Chem. 2009, 284, 8274-8282. 
(27) Obianyo, O.; Osborne, T. C.; Thompson, P. R. Kinetic mechanism of protein arginine methyltransferase. Biochemistry 2008, 47, 10420-10427.

(28) Gui, S.; Wooderchak-Donahue, W. L.; Zang, T.; Chen, D.; Daly\|, M. P.; Zhou, Z. S.; Hevel, J. M. Substrate-induced control of product formation by protein arginine methyltransferase 1. Biochemistry 2013, 52, 199-209.

(29) Rust, H. L.; Zurita-Lopez, C. I.; Clarke, S.; Thompson, P. R. Mechanistic studies on transcriptional coactivator protein arginine methyltransferase 1. Biochemistry 2011, 50, 33323345.

(30) Zhang, R.; Li, X.; Liang, Z.; Zhu, K.; Lu, J.; Kong, X.; Ouyang, S.; Li, L.; Zheng, Y. G.; Luo, C. Theoretical insights into catalytic mechanism of protein arginine methyltransferase 1. PLOS One 2013, 8, e72424.

(31) Chu, Y.; Li, G.; Guo, H. QM/MM MD and free energy simulations of the methylation reactions catalyzed by protein arginine methyltransferase PRMT3. Can. J. Chem. 2013, 91, 605612.

(32) Zhang, X.; Zhou, L.; Cheng, X. Crystal structure of the conserved core of protein arginine methyltransferase PRMT3. EMBO J. 2000, 19, 3509-3519.

(33) Gui, S.; Wooderchak, W. L.; Daly, M. P.; Porter, P. J.; Johnson, S. J.; Hevel, J. M. Investigation of the molecular origins of protein-arginine methyltransferase 1 (PRMT1) product specificity reveals a role for two conserved methionine residues. J. Biol. Chem. 2011, 286, 29118-29126.

(34) Gui, S.; Gathiaka, S.; Li, J.; Qu, J.; Acevedo, O.; Hevel, J. M. A remodeled protein arginine methyltransferase 1 (PRMT1) generates symmetric dimethylarginine. J. Biol. Chem. 2014, 289, 9320-9327.

(35) Eswar, N.; Webb, B.; Marti-Renom, M. A.; Madhusudhan, M. S.; Eramian, D.; Shen, M. Y.; Pieper, U.; Sali, A. Comparative protein structure modeling using modeller. Curr. Protoc. Protein. Sci. 2007, 50, 2.9.1-2.9.31.

(36) Altschul, S. F.; Madden, T. L.; Schaffer, A. A.; Zhang, J.; Zhang, Z.; Miller, W.; Lipman, D. J. Gapped blast and psi-blast: A new generation of protein database search programs. Nucleic Acids Res. 1997, 25, 3389-3402.

(37) Berman, H. M.; Westbrook, J.; Feng, Z.; Gilliland, G.; Bhat, T. N.; Weissig, H.; Shindyalov, I. N.; Bourne, P. E. The protein data bank. Nucleic Acids Res. 2000, 28, 235-242. 
(38) Troffer- Charlier, N.; Cura, V.; Hassenboehler, P.; Moras, D.; Cavarelli, J. Functional insights from structures of coactivator- associated arginine methyltransferase 1 domains. EMBO J. 2007, 26, 4391-4401.

(39) Laskowski, R. A. M., M. W.; Moss, D. S.; Thornton, J. M. J. Procheck: A program to check the stereochemical quality of protein structures. . J. Appl. Cryst. 1993, 26, 283-291.

(40) Wiederstein, M.; Sippl, M. J. Prosa-web: Interactive web service for the recognition of errors in three-dimensional structures of proteins. Nucleic Acids Res. 2007, 35, W407-410.

(41) Jorgensen, W. L. Yale University: New Haven, CT., 2004.

(42) Jorgensen, W. L.; Chandrasekhar, J.; Madura, J. D.; Impey, R. W.; Klein, M. L. Comparison of simple potential functions for simulating liquid water. J. Chem. Phys. 1983, 79, 926-935.

(43) Hornak, V.; Abel, R.; Okur, A.; Strockbine, B.; Roitberg, A.; Simmerling, C. Comparison of multiple amber force fields and development of improved protein backbone parameters. Proteins 2006, 65, 712-725.

(44) Wang, J.; Wolf, R. M.; Caldwell, J. W.; Kollman, P. A.; Case, D. A. Development and testing of a general amber force field. J. Comput. Chem. 2004, 25, 1157-1174.

(45) Gotz, A. W.; Williamson, M. J.; Xu, D.; Poole, D.; Le Grand, S.; Walker, R. C. Routine microsecond molecular dynamics simulations with amber on GPUs. 1. Generalized born. $J$. Chem. Theory Comput. 2012, 8, 1542-1555.

(46) Ryckaert, J.-P.; Ciccotti, G.; Berendsen, H. J. C. Numerical integration of the cartesian equations of motion of a system with constraints: Molecular dynamics of n-alkanes. J. Comput. Phys. 1977, 23, 327-341.

(47) Roe, D. R.; Cheatham, T. E. Ptraj and cpptraj: Software for processing and analysis of molecular dynamics trajectory data. J. Chem. Theory Comput. 2013, 9, 3084-3095.

(48) Pettersen, E. F.; Goddard, T. D.; Huang, C. C.; Couch, G. S.; Greenblatt, D. M.; Meng, E. C.; Ferrin, T. E. UCSF chimera - a visualization system for exploratory research and analysis. $J$. Comput. Chem. 2004, 25, 1605-1612.

(49) Miller, B. R.; McGee, T. D.; Swails, J. M.; Homeyer, N.; Gohlke, H.; Roitberg, A. E. Mmpbsa.Py: An efficient program for end-state free energy calculations. J. Chem. Theory Comput. 2012, 8, 3314-3321. 
(50) Zhou, R.; Xie, Y.; Hu, H.; Hu, G.; Patel, V. S.; Zhang, J.; Yu, K.; Huang, Y.; Jiang, H.; Liang, Z.; Zheng, Y. G.; Luo, C. Molecular mechanism underlying PRMT1 dimerization for sam binding and methylase activity. J. Chem. Inf. Model. 2015, 55, 2623-2632.

(51) Warshel, A.; Sharma, P. K.; Kato, M.; Xiang, Y.; Liu, H.; Olsson, M. H. M. Electrostatic basis for enzyme catalysis. Chem. Rev. 2006, 106, 3210-3235.

(52) Sadiq, S. K.; Coveney, P. V. Computing the role of near attack conformations in an enzyme-catalyzed nucleophilic bimolecular reaction. J. Chem. Theory Comput. 2015, 11, 316324.

(53) Bruice, T. C. A view at the millennium: The efficiency of enzymatic catalysis. Acc. Chem. Res. 2002, 35, 139-148.

(54) Sun, L.; Wang, M.; Lv, Z.; Yang, N.; Liu, Y.; Bao, S.; Gong, W.; Xu, R. M. Structural insights into protein arginine symmetric dimethylation by PRMT5. Proc. Natl. Acad. Sci. USA 2011, 108, 20538-20543.

(55) Gallivan, J. P.; Dougherty, D. A. Cation- $\pi$ interactions in structural biology. Proc. Natl. Acad. Sci. USA 1999, 96, 9459-9464. 
Graphical Abstract

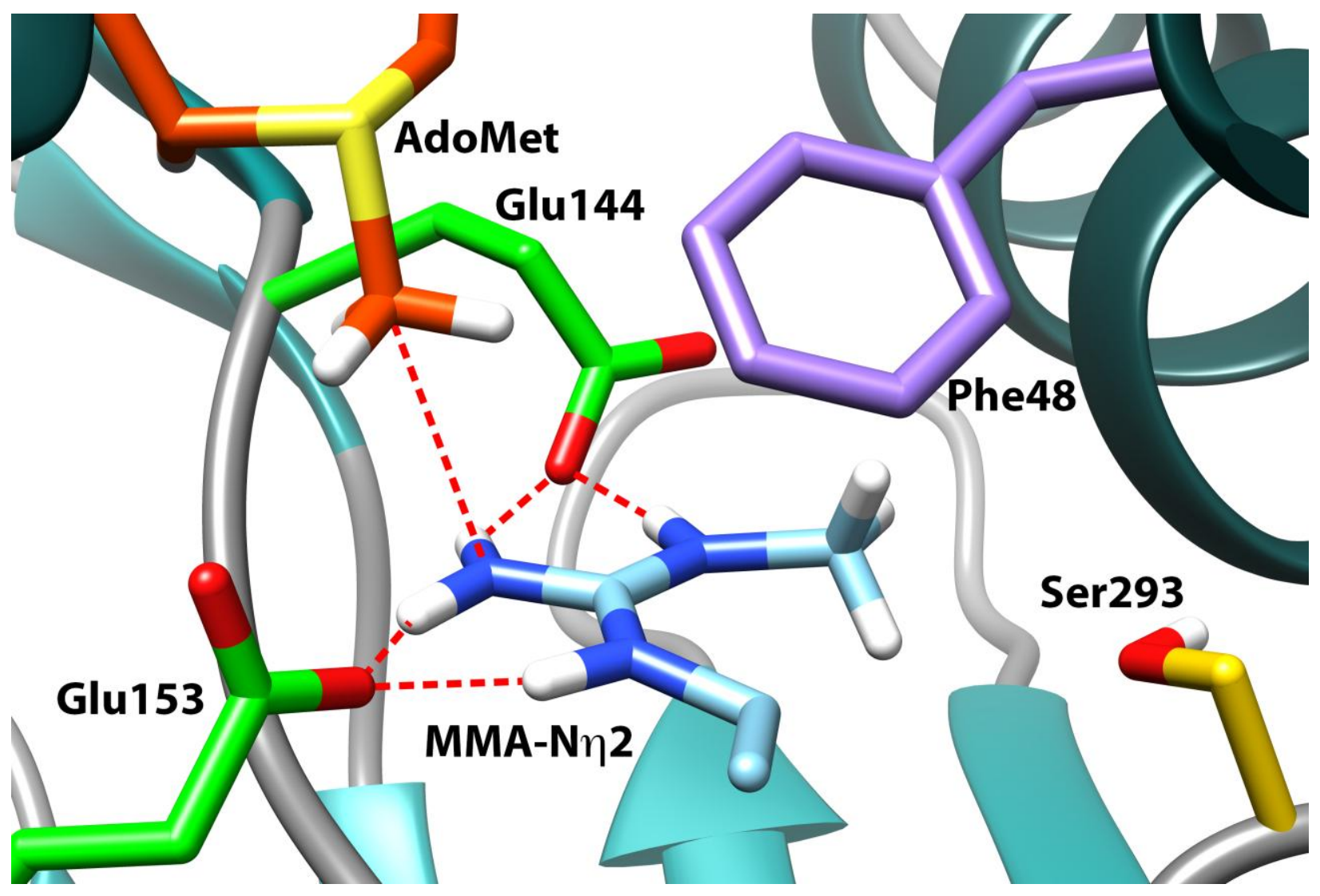

